\title{
In-situ muconic acid extraction reveals sugar consumption bottleneck in a xylose-utilizing Saccharomyces cerevisiae strain
}

Thomas Nicolaii ${ }^{1,2}$, Quinten Deparis ${ }^{1,2}$, María R. Foulquié-Moreno ${ }^{1,2^{*}}$ and Johan M. Thevelein ${ }^{1,2,3^{*}}$ (D)

\begin{abstract}
Background: The current shift from a fossil-resource based economy to a more sustainable, bio-based economy requires development of alternative production routes based on utilization of biomass for the many chemicals that are currently produced from petroleum. Muconic acid is an attractive platform chemical for the bio-based economy because it can be converted in chemicals with wide industrial applicability, such as adipic and terephthalic acid, and because its two double bonds offer great versatility for chemical modification.
\end{abstract}

Results: We have constructed a yeast cell factory converting glucose and xylose into muconic acid without formation of ethanol. We consecutively eliminated feedback inhibition in the shikimate pathway, inserted the heterologous pathway for muconic acid biosynthesis from 3-dehydroshikimate (DHS) by co-expression of DHS dehydratase from P. anserina, protocatechuic acid (PCA) decarboxylase (PCAD) from K. pneumoniae and oxygen-consuming catechol 1,2-dioxygenase (CDO) from C. albicans, eliminated ethanol production by deletion of the three PDC genes and minimized PCA production by enhancing PCAD overexpression and production of its co-factor. The yeast pitching rate was increased to lower high biomass formation caused by the compulsory aerobic conditions. Maximal titers of $4 \mathrm{~g} / \mathrm{L}, 4.5 \mathrm{~g} / \mathrm{L}$ and $3.8 \mathrm{~g} / \mathrm{L}$ muconic acid were reached with glucose, xylose, and a mixture, respectively. The use of an elevated initial sugar level, resulting in muconic acid titers above $2.5 \mathrm{~g} / \mathrm{L}$, caused stuck fermentations with incomplete utilization of the sugar. Application of polypropylene glycol 4000 (PPG) as solvent for in situ product removal during the fermentation shows that this is not due to toxicity by the muconic acid produced.

Conclusions: This work has developed an industrial yeast strain able to produce muconic acid from glucose and also with great efficiency from xylose, without any ethanol production, minimal production of PCA and reaching the highest titers in batch fermentation reported up to now. Utilization of higher sugar levels remained conspicuously incomplete. Since this was not due to product inhibition by muconic acid or to loss of viability, an unknown, possibly metabolic bottleneck apparently arises during muconic acid fermentation with high sugar levels and blocks further sugar utilization.

Keywords: Yeast cell factory, Muconic acid, Xylose, PDC negative, In-situ product removal, Lignocellulosic biomass, Glucose, ISPR, Pyruvate decarboxylase negative

*Correspondence: mrfoulquiem@gmail.com; johan.thevelein@kuleuven.be; johan.thevelein@novelyeast.com

1 Laboratory of Molecular Cell Biology, Institute of Botany and Microbiology, KU Leuven, Leuven-Heverlee, Belgium

Full list of author information is available at the end of the article

\section{Background}

The current shift from a fossil-based economy to a more sustainable, bio-based economy requires development of alternative production routes not only for fuels but also for the many chemicals that are currently produced from petroleum [1-3]. Over the last decade there has

(c) The Author(s) 2021. This article is licensed under a Creative Commons Attribution 4.0 International License, which permits use, sharing, adaptation, distribution and reproduction in any medium or format, as long as you give appropriate credit to the original author(s) and the source, provide a link to the Creative Commons licence, and indicate if changes were made. The images or other third party material in this article are included in the article's Creative Commons licence, unless indicated otherwise in a credit line to the material. If material is not included in the article's Creative Commons licence and your intended use is not permitted by statutory regulation or exceeds the permitted use, you will need to obtain permission directly from the copyright holder. To view a copy of this licence, visit http://creativeco mmons.org/licenses/by/4.0/. The Creative Commons Public Domain Dedication waiver (http://creativecommons.org/publicdomain/ zero/1.0/) applies to the data made available in this article, unless otherwise stated in a credit line to the data. 
been an increased interest in microbial production of muconic acid. Muconic acid is an attractive molecule for the bio-based economy because it can be converted into important platform chemicals, such as adipic acid and terephthalic acid [4]. It can also be used as a monomer in the production of heteropolymers [5] or homopolymers $[6,7]$ while retaining its double bonds, which allows for straightforward further chemical modification (Fig. 1).

Ideally, bio-based platform chemicals, such as muconic acid, are produced from second-generation substrates that are plentiful and cheap [8]. Lignocellulosic biomass is an excellent choice, but its use poses several challenges $[9,10]$. One major challenge is the efficient utilization of xylose [11, 12]. Two studies have reported the conversion of xylose into muconic acid, one using an $E$. coli co-culture [13] and the other an engineered S. cerevisiae lab strain [14]. Neither of these can be implemented in industrial practice due to their lack of robustness and inhibitor tolerance [9].

The most widely used de novo biosynthesis pathway for muconic acid starts with dehydroshikimate (DHS) as intermediate of the shikimate pathway. DHS is converted to protocatechuic acid (PCA), catechol and muconic acid, by combined expression of DHS dehydratase (DHSD), protocatechuic acid decarboxylase (PCAD) and catechol 1,2-dioxygenase (CDO). By further increasing availability of the shikimate pathway substrates, erythrose 4-phosphate (E4P) and phosphoenolpyruvate (PEP), and also enhancing DHS availability, Bui et al. obtained the highest muconic acid titer of $59 \mathrm{~g} / \mathrm{L}$ from glucose using $E$. coli as the host organism [15]. Although currently the highest reported muconic acid yields were obtained with bacteria as host organisms, they are not preferred for industrial production because of contamination risks and their sensitivity to low $\mathrm{pH}$, which complicates downstream processing of the muconic acid produced [16]. Industrial strains of the yeast S. cerevisiae are considered a more preferred alternative because of their high tolerance to low $\mathrm{pH}$, general robustness and high stress tolerance in industrial conditions.

The first study implementing S. cerevisiae as a host organism reported a very low titer of $1.56 \mathrm{mg} / \mathrm{L}$ and a yield of $0.78 \mathrm{mg} / \mathrm{g}$ [17]. In subsequent studies two modifications were applied to enhance titer and yield [18]. The feedback insensitive 3-deoxy-D-arabino-heptolusonate7-phosphate (DAHP) synthase enzymes, $A R O 3^{K 222 L}$ and $A R O 4^{K 229 L}$ were expressed to increase flux into the shikimate pathway [19-23], as well as transketolase TKL1 to enhance E4P availability [20, 21, 23-25].

Other studies have used various heterologous genes to achieve higher muconic acid titers [21-25]. The PCAD enzyme received particular attention because of the high PCA accumulation. In general the K. pneumoniae PCAD enzyme has been preferred and is encoded by the native $\mathrm{BCD}$ cluster. While the $\mathrm{C}$ isoform (aroY-C) encodes the actual PCAD enzyme, the $\mathrm{B}$ isoform (aroY-B) has a supportive function by production of the prenylated flavin mononucleotide (prFMN) cofactor [26]. Another PCAD homologue $\left(\operatorname{aro} Y-C^{i s o}\right)$ was shown to be less oxygen sensitive displaying higher activity under oxygen-limited conditions $[17,27]$. The gene PAD1 in S. cerevisiae is a paralog to aro $Y-B$ and its overexpression led to increased PCAD activity [28]. Increasing the copy number of coexpressed aroY-B and aroY- $C^{i s o}$ enhanced PCAD activity and muconic acid production, confirming the rate-limiting character of PCAD [25, 29]. Alternative prFMNindependent PCAD enzymes were shown to be superior to the co-expression of aro $Y-B$ and aro $Y$ - $C^{i s o}$ in yeast strains that do not express PAD1 [20,30], but it remains unclear whether they support higher PCAD activity than the co-expression of $P A D 1$ and aroY-C ${ }^{i s o}$. Recently, Wang et al. expressed a heterologous muconic acid production

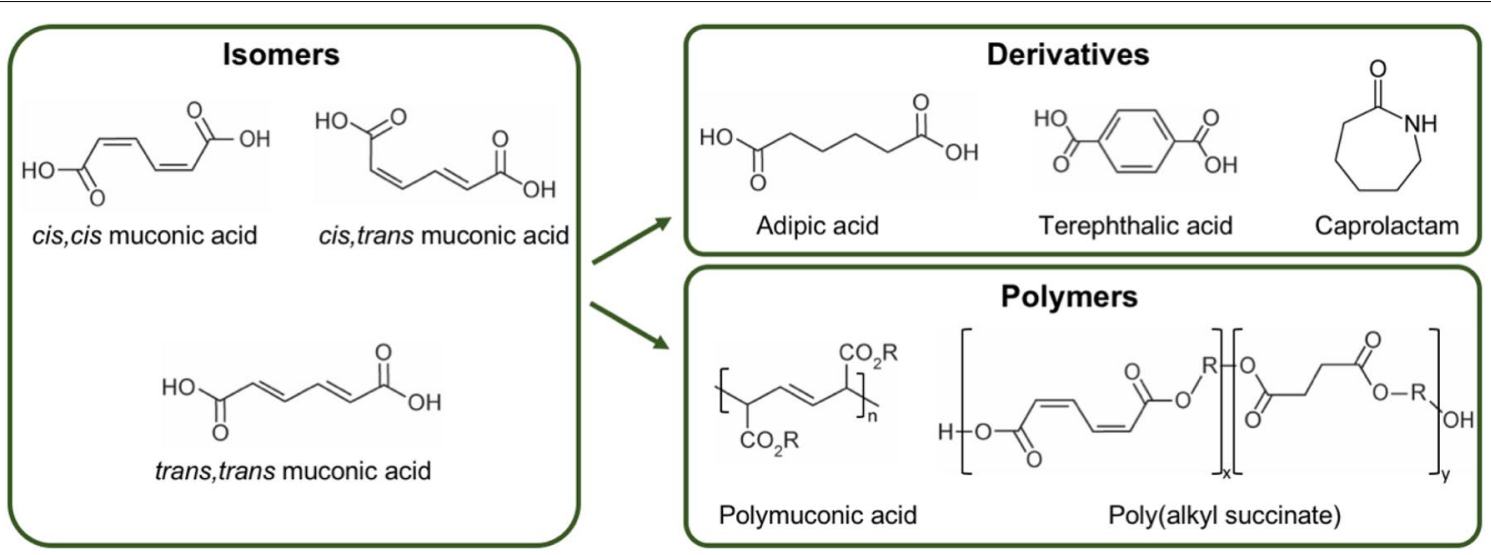

Fig. 1 Chemical structures of muconic acid isomers, derivatives and polymers 
pathway in S. cerevisiae and enhanced the performance of the strain through biosensor-aided and subsequent rational genomic engineering approaches. This study also showed that muconic acid was toxic at concentrations of $5 \mathrm{~g} / \mathrm{L}$ and higher when the $\mathrm{pH}$ was not controlled. Ethanol production was lowered by optimizing the feed solution and feeding rate in a controlled fed-batch fermentation. These optimizations combined with maintenance of the $\mathrm{pH}$ at 6 to minimize toxicity, led to a titer of $20.8 \mathrm{~g} / \mathrm{L}$ and a yield of $66.2 \mathrm{mg} / \mathrm{g}$ muconic acid, which is the highest titer and yield for muconic acid production with S. cerevisiae reported to date.

Up until now, none of the yeast strains used for muconic acid production completely lacked ethanol production, likely a major cause of the low yields obtained. Ethanol production can be eliminated by deleting the three pyruvate decarboxylase (PDC) encoding genes $P D C 1, P D C 5$ and PDC6. However, this severely compromises growth and metabolism of the yeast because of shortage in cytosolic acetyl-CoA [31, 32]. This problem can be addressed to some extent by supplying a C2 compound such as ethanol in the medium, or by a partial internal deletion of the MTH1 gene [33-38]. However, neither approach overcomes the problem completely and elimination of ethanol production to enhance yield thus remains challenging.

A universal stress factor for cell factories is toxicity of the end-product, because it has to be produced at a high titer to ensure efficient downstream processing for the economic viability of the industrial process. While increasing the $\mathrm{pH}$ of the fermentation medium results in lower muconic acid toxicity [39], it adds complexity to the muconic acid recovery process and compromises economic feasibility. One way to circumvent this problem is in-situ product removal (ISPR) of muconic acid during the production process. Various methodologies exist for extracting carboxylic acids [40] and more specifically muconic acid [41-45]. Although the suggested solvents are supposedly biocompatible, there are no published results on the use of these solvents in combination with host organisms producing muconic acid.

In this work we have engineered an industrial secondgeneration yeast strain with efficient xylose consumption and high inhibitor tolerance for muconic acid production. We eliminated for the first time ethanol production completely, strongly minimized production of the PCA intermediate and optimized the strain for maximal titer, productivity and yield. With this strain we produced the highest reported titer of muconic acid in batch fermentations to date. To overcome stuck fermentations when using a high initial sugar level, presumed to be due to muconic acid toxicity at low medium $\mathrm{pH}$, we developed ISPR with polypropylene glycol 4000 (PPG) as solvent.
This surprisingly revealed an unexpected, possibly metabolic bottleneck in utilization of high sugar levels arising during muconic acid production and unrelated to its toxicity.

\section{Materials and methods \\ Yeast strains and plasmids}

The yeast strains and plasmids used and constructed in this work are shown in Tables 1 and 2, respectively.

\section{Growth media and culturing conditions}

Propagation of yeast cells was performed in YPD medium containing $1 \%$ yeast extract, $2 \%$ bacteriological peptone and $2 \%$ glucose at $30{ }^{\circ} \mathrm{C}$ in a rotary shaker (200 rpm). For propagation of $p d c$-negative strains, glucose was replaced by $2 \%$ glycerol and $2 \%$ ethanol as carbon source. In order to have sufficient aeration during growth of $p d c$-negative strains, propagation was performed in shake flasks $(100 \mathrm{~mL}$ or $300 \mathrm{~mL})$ under constant shaking (200 rpm). Media were supplemented with $1.5 \%$ bacto $^{\mathrm{TM}}$ agar to obtain solid nutrient agar plates. Transformants were selected on solid agar plates supplemented with either one or a combination of the following antibiotics: $200 \mathrm{mg} / \mathrm{L}$ geneticin, $300 \mathrm{mg} / \mathrm{L}$ hygromycin B or $200 \mathrm{mg} / \mathrm{L}$ nourseothricin. In case of $p d c$-negative strains, only $20 \%$ of the abovementioned amounts of antibiotics was used. For storage of strains for longer periods of time, 30\% glycerol was added to YP medium in sterile screw-cap tubes which were stored at $-80^{\circ} \mathrm{C}$. Cells were plated out and incubated at $30{ }^{\circ} \mathrm{C}$ when taken out of the stock. Maintenance and selection of plasmids in E. coli (TOP10) was performed in lysogeny broth (LB) medium supplemented with $100 \mathrm{mg} / \mathrm{L}$ ampicillin or $50 \mathrm{mg} / \mathrm{L}$ kanamycin. Solid LB plates contained $1.5 \%$ bacto $^{\mathrm{TM}}$ agar.

\section{Genomic DNA and plasmid extraction, PCR}

Genomic DNA of yeast cells was extracted by phenol/chloroform/isoamyl-alcohol (25:24:1) and precipitated with ethanol. PCR was performed with low-fidelity Standard Taq or TaqE polymerase to test for positive transformants or with high-fidelity polymerase Q5 for sequencing and cloning.

Plasmids were extracted from E. coli using the commercial Nucleospin Plasmid EasyPure kit (MachereyNagel) after which digestion with relevant restriction enzymes was performed. Cloned plasmids were transformed in E. coli and transformants selected on LB agar plates containing the appropriate antibiotic. Transformants were checked by colony-PCR for presence of the correct construct using low-fidelity Standard Taq or TaqE polymerase. Positive transformants were grown overnight in liquid LB medium, supplemented with the appropriate antibiotic. Plasmids were submitted for 
Table 1 Strains used and constructed in this study

\begin{tabular}{|c|c|c|}
\hline Yeast strains & $\begin{array}{l}\text { Description } \\
\text { Genotype }\end{array}$ & Source \\
\hline W303 & MATa can1-100 ade2-1 his3-11,15 leu2-3 trp1-1 ura3-1 & MCB, KU Leuven \\
\hline GSE16-T18HAA1 & $\begin{array}{l}\text { Industrial } 2 \mathrm{G} \text { xylose-fermenting and inhibitor-tolerant strain } \\
\text { GSE16-T18 with C.1517 G>A mutation in both copies of HAA1 }\end{array}$ & [46] \\
\hline TN7 & GSE16-T18HAA1 aro3 $\triangle A R O 4^{K 229 L}$ & This study \\
\hline TN8 & TN7 MTH1 $1^{-M 41-T 78}$ & This study \\
\hline TN9 & $\begin{array}{l}\text { TN8 pdc1::MApw } \\
\text { MApw being TDH3p - HQD2 }{ }_{C a}-A D H 1 t, A D H 1 p-\text { aroY-Ciso }-A D H 2 t \text {, TEF1p - aroZ } Z_{\text {Pan }}-C Y C 1 t\end{array}$ & This study \\
\hline TN10 & $\begin{array}{l}\text { TN9 IS2.1:: FBA1 p - PAD1 - ADH1t } \\
\text { IS2.1, integrative site NC_001134:g.140631^14632 (yeast 2015) }\end{array}$ & This study \\
\hline TN5 & $\begin{array}{l}\text { TN10 pdc6::Mapw } \\
\text { Mapw being TDH3p - HQD2 }{ }_{C a}-A D H 1 t, A D H 1 p-\text { aroY-Ciso }-A D H 2 t \text {, TEF1p - aroZ } Z_{\text {Pan }}-C Y C 1 t\end{array}$ & This study \\
\hline TN6 & 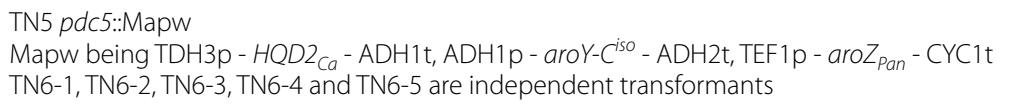 & This study \\
\hline TN14 & $\begin{array}{l}\text { TN6-1 IS16.2:: PGK1p - AGDC1 - TPS1t } \\
\text { IS16.2, integrative site NC_001 148:g.621736^621737 (yeast 2015) }\end{array}$ & $\begin{array}{l}\text { This study } \\
{[20]}\end{array}$ \\
\hline TN15 & $\begin{array}{l}\text { TN6-1 IS16.2:: PGK1p - TaGDC1 - TPS1t } \\
\text { IS16.2, integrative site NC_001 148:g.621736^621737 (yeast 2015) }\end{array}$ & $\begin{array}{l}\text { This study } \\
{[20]}\end{array}$ \\
\hline TN16 & $\begin{array}{l}\text { TN6-1 } \operatorname{aroY}-C^{\text {iso }}(\mathrm{WT}) \\
\text { aroY-C } \text { iso, } 1218 \mathrm{~V}_{\text {in TN6-1 }} \text { at PDC5 locus to wild type aroY-C } C^{\text {iso }}\end{array}$ & This study \\
\hline TN22 & $\begin{array}{l}\text { TN6-1 IS16.2:: PGK1p-aroY-Ciso }- \text { TKL1t } \\
\text { IS16.2, integrative site NC_001148:g.621736^621737 (yeast 2015) }\end{array}$ & This study \\
\hline
\end{tabular}

Sanger sequencing at the Genetic Service Facility of the VIB.

\section{Gene synthesis and cloning}

The ORF of the following heterologous genes was codon optimized and ordered at IDT: DHSD from Podospora anserina (aroZ $Z_{\text {Pan }}$ PA_5_5120; GeneBank Accession number CAD60599), CDO from Candida albicans (HQD2 ${ }_{\mathrm{Ca}}$; GeneBank Accession number KHC86777), PCAD from Klebsiella pneumoniae (aroY$C^{\text {iso }}$; GeneBank Accession number AB479384), Arxula adeninivorans (AGDC1; GeneBank Accession number SJN60119.1) and Talaromyces atroroseus (TaGDC1, GeneBank Accession number XP 020118381.1), and flavin prenyltransferase from $K$. pneumoniae (aroY-B, GeneBank Accession number AAY57854). Using the high-fidelity polymerase Q5 (New England Biolabs) the genes obtained were PCR-amplified with a low cycle number to prevent accidental mutations. Next, the amplicons were purified with the commercial Wizard SV Gel and PCR clean-up (Promega) kit and cloned into the respective vectors using Gibson assembly master mix (New Englands Biolabs). The ORF of the $P A D 1$ gene was amplified from the genome of the W303 lab strain.

\section{Strain construction}

Yeast transformation was performed either by Lithiumacetate protocol or electroporation [48, 49]. Deletion of ARO3 was performed with a KanMX marker, flanked with attB and attP recombination sites. Removal of the integrated KanMX marker in the positive transformants was achieved by transformation of the PhiC31 integrase vector, which recombines attB and attP, leaving an attL scar. The remaining genomic adjustments were carried out with the CRISPR/Cas9 genome editing tool. Yeast cells were transformed with the p51 plasmid, carrying the Cas9 enzyme, and precultured for a second transformation in YP media with the appropriate carbon source and geneticin. During the second transformation, gRNA plasmid and linear donor DNA (amplified from the donor DNA backbone with high-fidelity Q5 polymerase) were transformed into the yeast cells and the transformants were selected for presence of both Cas9 and gRNA plasmids. The PDC1 gene was replaced by a stepwise approach in which the ORF was first replaced by a NatMX marker, flanked with G1 gRNA recognition sites that were targeted in a subsequent transformation with the G1 gRNA. Other genomic adjustments and knockins were conducted by a one-step approach, directly targeting and replacing genomic DNA. Plasmid loss was achieved by $3 \times$ serial transfer in YP medium, after 
Table 2 Plasmids used and constructed in this study

\begin{tabular}{|c|c|c|}
\hline Plasmid & Description & Source \\
\hline \multicolumn{3}{|l|}{ Generic plasmids and plasmid backbones } \\
\hline pJET1.2-B-KanMX-P & $\begin{array}{l}\text { pMB1 ori (E. coli) } \\
\text { Backbone for amplification of KanMX marker flanked by attB and attP } \\
\text { (attB/P) }\end{array}$ & MCB, KU Leuven \\
\hline pTOPO-G1-NAT-G1 & $\begin{array}{l}\text { pMB1 ori (E. coli) } \\
\text { Backbone for amplification of NatMX marker flanked by attB/P and G1 } \\
\text { recognition site }\end{array}$ & MCB, KU Leuven \\
\hline PhiC31NAT & $\begin{array}{l}\text { ColE1 ori (E. coli) and } 2 \text { micron ori (S. cerevisiae, multi-copy), encodes } \\
\text { PhiC31 integrase and NatMX marker }\end{array}$ & MCB, KU Leuven $[47,47]$ \\
\hline p426KanMX & $\begin{array}{l}\text { pMB1 ori (E. coli) and } 2 \text { micron ori (S. cerevisiae, multi-copy), KanMX } \\
\text { marker } \\
\text { Backbone for construction of donor DNA }\end{array}$ & MCB, KU Leuven \\
\hline p426hph & $\begin{array}{l}\text { pMB1 ori (E. coli) and } 2 \text { micron ori (S. cerevisiae, multi-copy), hph marker } \\
\text { Backbone for construction of Mapw and donor DNA }\end{array}$ & MCB, KU Leuven \\
\hline pBEVYhph & $\begin{array}{l}\text { ColE1 ori (E. coli) and } 2 \text { micron ori (S. cerevisiae, multi-copy), hph marker } \\
\text { Backbone for construction of Mapw }\end{array}$ & MCB, KU Leuven \\
\hline pTEF-Cas9-KanMX (p51) & $\begin{array}{l}\text { pBR322 ori (E. coli) and CEN ori (single copy), vector backbone p414- } \\
\text { TEF1p-Cas9-CYC1t with auxotrophic marker replaced by KanMX }\end{array}$ & MCB, KU Leuven \\
\hline pgRNA-uni-NAT (p59) & $\begin{array}{l}\text { pBR322 ori (E. coli) and } 2 \text { micron ori (S. cerevisiae, multi-copy) } \\
\text { gRNA plasmid backbone with NatMX marker }\end{array}$ & MCB, KU Leuven \\
\hline pgRNA-uni-hph (p58) & $\begin{array}{l}\text { pBR322 ori (E. coli) and } 2 \text { micron ori (S. cerevisiae, multi-copy) } \\
\text { gRNA plasmid backbone with hph marker }\end{array}$ & MCB, KU Leuven \\
\hline p426hph-IS2.1 & Multi copy, homologous regions for IS2.1 with hph marker & MCB, KU Leuven \\
\hline p426hph-IS16.2 & p426hph backbone with homologous regions for IS16.2 & MCB, KU Leuven \\
\hline \multicolumn{3}{|l|}{ Guide RNA plasmids } \\
\hline pgRNA-uni-hph-G1-G2 & $\begin{array}{l}\text { P58 backbone with gRNA sequence targeting G1 (GGCTGATTTTCGCAG } \\
\text { TTCGG) and G2 (GGATGAGAATCTGACAAAGGG) }\end{array}$ & MCB, KU Leuven \\
\hline $\mathrm{p} 58-A R O 4$ & p58 backbone with gRNA targeting ARO4 (TTCATGGGTGTTACTAAGCA) & This study \\
\hline p58-MTH1 & $\begin{array}{l}\text { p58 backbone with gRNA sequence targeting MTH1 (CTAGCTCTATCA } \\
\text { GTGTACTC) }\end{array}$ & This study \\
\hline p58-PDC5 & $\begin{array}{l}\text { p58 backbone with two gRNA sequences targeting PDC5 (AGCATCCAA } \\
\text { CAATTTTTGCA and GATAAGCTTTATGAAGTCAA) }\end{array}$ & This study \\
\hline p58-PDC6 & $\begin{array}{l}\text { p58 backbone with two gRNA sequences targeting PDC6 (CTATCGAAA } \\
\text { AGCTGATTCAT and GCTGATTTGATCCTTTCGGT) }\end{array}$ & This study \\
\hline p59-aroY-Ciso,1218V & $\begin{array}{l}\text { p59 backbone with gRNA sequence targeting aroY-Ciso,1218V (TTAGAT } \\
\text { CCCGCTATCTACGT) }\end{array}$ & This study \\
\hline p59-attL & Multi copy, gRNA for attL site & This study \\
\hline p59-IS2.1 & $\begin{array}{l}\text { p59 backbone with gRNA sequence targeting the IS2.1 site (ATCAAC } \\
\text { CACAGTGAACGCCG) }\end{array}$ & This study \\
\hline p59-IS16.2 & $\begin{array}{l}\text { p59 backbone with gRNA sequence targeting the IS16.2 site (GTAGAA } \\
\text { TAAGTGTTTCGGAT) }\end{array}$ & This study \\
\hline \multicolumn{3}{|l|}{ Plasmids containing expression cassettes } \\
\hline p426KanMX-PAD1 & $\begin{array}{l}\text { p426KanMX backbone with the expression cassette FBA1p; PAD1; } \\
\text { ADH2t }\end{array}$ & This study \\
\hline p426hph-IS2.1-PAD1 & $\begin{array}{l}\text { p426hph-IS2.1 backbone with the expression cassette FBA 1 p; PAD1; } \\
\text { ADH2t flanked by the homologous regions for genomic integration } \\
\text { at IS2.1 }\end{array}$ & This study \\
\hline p426KanMX-aroY-B & $\begin{array}{l}\text { p426KanMX backbone with the expression cassette FBA1p; aroY-B; } \\
\text { ADH2t }\end{array}$ & This study \\
\hline p426KanMX-DHSD & $\begin{array}{l}\text { p426KanMX backbone with the expression cassette TEF1p; aro } Z_{\text {pani }} \\
\text { CYC1t }\end{array}$ & This study \\
\hline pBEVYhph-CDO-PCAD(aroY-Ciso) & $\begin{array}{l}\text { pBEVYhph backbone with the expression cassettes TDH3p - } \mathrm{HQD}_{\mathrm{Ca}^{-}} \\
\text {ADH1t, ADH1p - aroY- } \mathrm{C}^{\text {iso }}-\mathrm{ADH} 2 \mathrm{t} \\
\left.\text { Together with p426KanMX-DHSD constitutes pMApw(aroY- } \mathrm{C}^{\text {iso }}\right)\end{array}$ & This study \\
\hline pBEVYhph-CDO-PCAD(aroY-Ciso)-DHSD (pMApw) & $\begin{array}{l}\text { pBEVYhph backbone with the expression cassettes TDH3p - HQD2 }{ }_{C a}- \\
\text { ADH1t, ADH1p - aroY- } \mathrm{C}^{\text {iso }}-\mathrm{ADH} 2 \mathrm{t}, \mathrm{TEF} 1 \mathrm{p}-\text { aro }_{\text {pan }}-\mathrm{CYC} 1 \mathrm{t} \\
\text { Contains the muconic acid pathway (MApw) }\end{array}$ & This study \\
\hline
\end{tabular}


Table 2 (continued)

\begin{tabular}{|c|c|c|}
\hline Plasmid & Description & Source \\
\hline pMApw-PDC1 & $\begin{array}{l}\text { pMApw backbone with homologous regions for genomic integration } \\
\text { at the PDC1 locus }\end{array}$ & This study \\
\hline pMApw-PDC5 & $\begin{array}{l}\text { pMApw backbone with homologous regions for genomic integration } \\
\text { at the PDC5 locus }\end{array}$ & This study \\
\hline pMApw-PDC6 & $\begin{array}{l}\text { pMApw backbone with homologous regions for genomic integration } \\
\text { at the PDC6 locus }\end{array}$ & This study \\
\hline p426hph-IS16.2-AGDC1 & $\begin{array}{l}\text { p426hph-IS16.2 backbone with the expression cassette PGK1p - AGDC1 } \\
\text { - TPS1t flanked by the homologous regions for genomic integration } \\
\text { at IS16.2 }\end{array}$ & This study \\
\hline p426hph-IS16.2-TaGDC1 & $\begin{array}{l}\text { p426hph-IS16.2 backbone with the expression cassette PGK1p - TaGDC1 } \\
\text { - TPS1t flanked by the homologous regions for genomic integration } \\
\text { at IS16.2 }\end{array}$ & This study \\
\hline p426hph-IS16.2-aroY-Ciso & $\begin{array}{l}\text { p426hph-IS16.2 backbone with the expression cassette PGK1p - aroY- } \\
\text { Ciso - TPS1t flanked by the homologous regions for genomic integra- } \\
\text { tion at IS16.2 }\end{array}$ & This study \\
\hline p426hph-IS16.3-ARO3 $3^{K 222 L}-A R O 4^{K 229 L}$ & $\begin{array}{l}\text { p426hph-IS16.3 backbone with the expression cassettes PGK1p - } \\
\text { ARO3 }{ }^{\text {K222L }} \text { - TPS1t, TEF1p; ARO4 }{ }^{K 222 L} \text { - ELO2t flanked by the homolo- } \\
\text { gous regions for genomic integration at IS16.3 }\end{array}$ & This study \\
\hline
\end{tabular}

which single cells were isolated and spotted on solid agar medium containing relevant antibiotics to screen for loss of resistance.

\section{Muconic acid fermentations and HPLC analysis}

Yeast cells were propagated in YP medium with the appropriate carbon source till stationary phase. Cells were washed with sterile water and inoculated at different $\mathrm{OD}_{600}$ levels, as indicated, in $300 \mathrm{~mL}$ shake flasks with a total of $40 \mathrm{~mL}$ YP medium. Technical duplicates or biological triplicates were used for each strain, with the standard deviation indicated. Samples for $\mathrm{pH}$ measurements and metabolite analysis were taken at various time points. For HPLC analysis, the supernatant was separated from the cells by centrifugation $(12,000 \mathrm{rpm}, 2 \mathrm{~min})$ and analysed with a Biorad Aminex HPX $300 \times 7.8 \mathrm{~mm}$ column. From the supernatant, $10 \mu \mathrm{L}$ was injected in a $5 \mathrm{mM} \mathrm{H}_{2} \mathrm{SO}_{4}$ mobile phase with a flow rate of $0.65 \mathrm{~mL} /$ min, while the column temperature was maintained at $60{ }^{\circ} \mathrm{C}$. Protocatechuic acid, catechol and muconic acid were detected at $270 \mathrm{~nm}$ using the UV detector (SPD20A, Shimadzu). Glucose, xylose and ethanol were quantified using the RID detector (RID-20A, Shimadzu). In both cases standard curves were used.

\section{Whole genome sequence analysis}

Genomic DNA was extracted using the MasterPure Yeast DNA purification kit (Epicentre, Lucigen). Strains TN5 (Samplen220) and TN6-1 (Samplen221) were sent to BGI (China) for next-generation sequencing with Illumina (HiSeq2500) using a 500 bp insert library and pair-end reads of 125 bp. Strains TN6-2 (Samplen267), TN6-3
(Samplen268), TN6-4 (Samplen269) and TN6-5 (Samplen270) were sent to Eurofins Genomics (Germany) for next-generation sequencing with Illumina (HiSeq2500) using a $500 \mathrm{bp}$ insert library and pair-end reads of $150 \mathrm{bp}$. The reads were mapped against the S288c reference genome (R64-2,1; SGD) using bowtie 2 [50]. Coverage of the TN5 and the different TN6 strains was determined using samtools [51] and compared to the GSE16T18HAA1 strain using NGSEP [52]. Variants were called using NGSEP and SNP frequency was determined using in-house scripts. The data have been deposited with links to the BioProject accession number PRJNA699459 in the NCBI BioProject database (https://www.ncbi.nlm.nih. giv/bioproject/).

\section{Determination of distribution coefficient (Kd) for PPG}

To represent the fermentation conditions, a YPD medium was spiked with muconic acid and adjusted to a $\mathrm{pH}$ of $4.25 \pm 0.05$. Then $20 \%(\mathrm{v} / \mathrm{v})$ of the solvent was added. The mixtures were shaken vigorously at $30{ }^{\circ} \mathrm{C}$ for $30 \mathrm{~min}$ to reach extraction equilibrium. Next, the samples were centrifuged (14,000 rpm, $3 \mathrm{~min})$ and the concentration of muconic acid in the aqueous phase was determined by HPLC analysis. Using the equation below, where $c_{\text {org }}$ and $\mathrm{c}_{\mathrm{aq}}$ are the concentrations of muconic acid in the organic phase and in the aqueous phase, respectively, we calculated a distribution coefficient $(\mathrm{Kd})$ of 5.78 for PPG.

$$
K d=\frac{c_{o r g}}{c_{a q}}
$$

The $\mathrm{c}_{\text {org }}$ was calculated based on the concentration of muconic acid in the aqueous phase before and after 
extraction, while taking into account the applied volumetric solvent ratio of $20 \%(\mathrm{v} / \mathrm{v})$.

\section{Results}

Construction of a xylose-utilizing pdc- S. cerevisiae strain with efficient muconic acid production

To construct an industrially relevant yeast strain for muconic acid production from lignocellulosic biomass, we used the diploid $S$. cerevisiae second-generation bioethanol strain GSE16-T18 HAA1 as a host strain [46]. First, we focused on improving the flux in the central shikimate pathway, used for production of aromatic amino acids, to improve the availability of the DHS intermediate that will serve as substrate for the heterologous muconic acid pathway. For that purpose, we deleted $A R O 3$ and introduced the point mutation $\mathrm{K} 229 \mathrm{~L}$ in $A R O 4$ to overcome the feedback inhibition in the shikimate pathway (Fig. 2), which resulted in strain TN7.

Next, we constructed a plasmid with the heterologous pathway for muconic acid production (MApw) by co-expression of three codon-optimized heterologous enzymes: DHSD from $P$. anserina (aro $Z_{\text {Pan }} /$ PA_5_5120), PCAD from K. pneumoniae (aroY-C ${ }^{i s o}$ ) and CDO from C. albicans $\left(H Q D 2_{C a}\right)$ under control of the TEF1, $A D H 1$ and $T D H 3$ constitutive promoter, respectively. We introduced the plasmid expressing the MApw into the TN7 strain, which resulted in high production of the PCA intermediate and a transient production of minor levels of muconic acid due to its consumption under aerobic conditions (Additional file 1).

Next we eliminated ethanol production by deleting both copies of the PDC1, PDC5, PDC6 genes. For that purpose, we first deleted part of the MTH1 gene

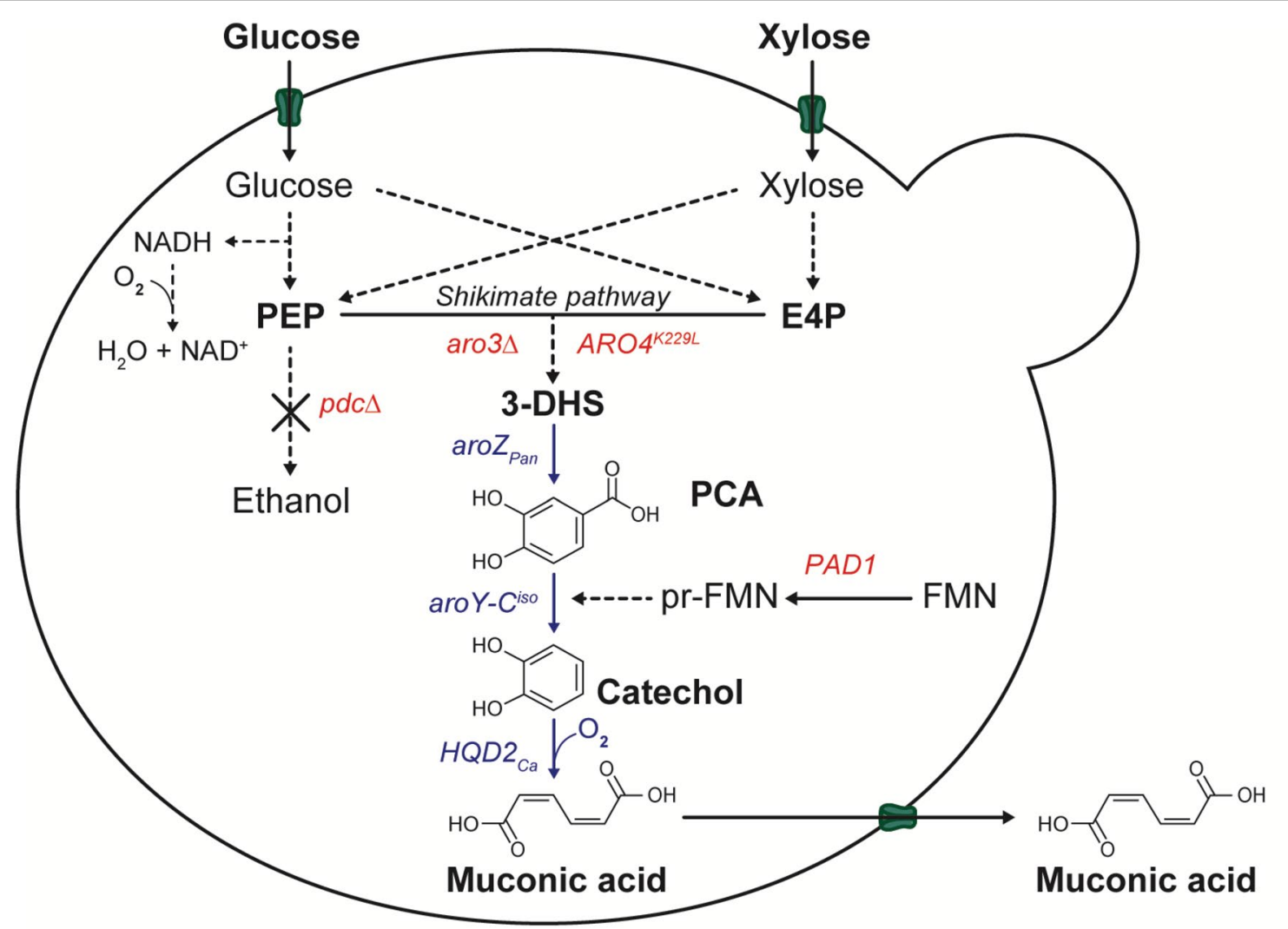

Fig. 2 Pathways used to produce muconic acid from glucose and xylose. Glucose and xylose are converted after uptake into phosphoenolpyruvate (PEP) and erythrose-4-phosphate (E4P) by the glycolytic and pentose-phosphate pathways. The two compounds are combined to enter the shikimate pathway and produce 3-dihydroshikimate (DHS), which is then converted using the bacterial heterologous pathway for muconic acid biosynthesis (MApw) into protocatechuic acid (PCA), catechol and muconic acid. The MApw consists of a DHS dehydratase from Podospora anserina (aroZ $Z_{\text {pan }}$ ), PCA decarboxylase (PCAD) from K. pneumoniae (aroY-C $C^{i s o}$ ) and catechol dioxygenase (CDO) from C. albicans ( $H Q D 2_{C a}$ ). Feedback inhibition of aromatic amino acids acting on Aro3 and Aro4, has been relieved by deletion of Aro3 and site-specific mutagenesis of Aro4 (ARO4 ${ }^{K 229 L}$ ). Overexpression of PAD1 stimulates production of prenylated flavine mononucleotide (prFMN), a co-factor of the protocatechuic acid decarboxylase (PCAD) enzyme encoded by aroY- $C^{\text {iso }}$. To further enhance muconic acid production, the synthesis of ethanol was abolished by deletion of the pyruvase decarboxylase (PDC) genes $(p d c \Delta)$. Muconic acid is exported from the cells by one or more unknown carriers 
$\left(M T H 1^{\Delta M 41-T 78}\right)$, which is known to alleviate glucose repression and restore growth on glucose in $p d c$ - strains $[35,38]$. This resulted in strain TN8 (TN7 $\left.M T H 1^{\Delta M 41-T 78}\right)$.

Next, we integrated the MApw in the genome at the $P D C 1$ locus, replacing the two copies of the $P D C 1$ gene, yielding strain TN9 (TN8 pdc1::MApw/pdc1::MApw). Since this strain still produced large amounts of PCA and only minor amounts of muconic acid (Additional file 2), we increased PCAD activity by expression of PAD1 or aro $Y-B$ on a multi-copy plasmid. Since expression of $P A D 1$ resulted in a higher muconic acid level (Additional file 3), we next introduced two copies of PAD1 in the genome under control of the $F B A 1$ promoter, resulting in the strain TN10 (TN9 NC_001134.8:g.140631_140632in sPAD1). TN10 showed higher muconic acid production compared to TN9 (Additional file 4). We then integrated two copies of the MApw at the PDC6 locus to create strain TN5 (TN10 $p d c 6:: \mathrm{MApw} / p d c 6:: \mathrm{MApw})$. TN5 showed higher production of PCA and muconic acid compared to TN10 (Additional file 5). In these fermentations the level of catechol was always undetectable, which shows that the CDO enzyme confers highly efficient conversion of catechol into muconic acid (Additional files 3, 5).

Finally, we replaced the two PDC5 copies with the MApw in TN5 in order to abolish ethanol production completely, resulting in strain TN6 (TN5 $p d c 5:: \mathrm{MApw} / p d c 5:: \mathrm{MApw})$. This $p d c$-negative ( $p d c-)$ strain was selected on solid nutrient medium containing glucose and ethanol as a $\mathrm{C} 2$ source to support cytosolic acetyl-CoA synthesis. The TN6 transformants showed large differences in colony size, suggesting the occurrence of additional background mutations improving e.g. glucose tolerance and utilization. Several TN6 transformants with large colony size were evaluated in shake flask cultures with glucose and ethanol as carbon sources for the muconic acid production. In this experiment, the strains were inoculated at a low $\mathrm{OD}_{600}$ of 0.1 to allow more extensive growth, which also served as a selection tool to obtain the best transformants (Fig. 3A). The screened isolates showed different rates of glucose utilization, PCA and muconic acid production. They also consumed the ethanol at different rates. One isolate, TN6-1, clearly showed superior behaviour: a rapid glucose utilization and the highest PCA and muconic acid production. Moreover, he TN6-1 isolate showed a strongly transient accumulation of PCA, reaching a maximum titer of $490 \mathrm{mg} / \mathrm{L}$ at $96 \mathrm{~h}$ and declining to $180 \mathrm{mg} / \mathrm{L}$ at $168 \mathrm{~h}$, which correlated to a further increase in muconic acid titer reaching $960 \mathrm{mg} / \mathrm{L}$ at $168 \mathrm{~h}$.

The three best performing isolates, TN6-1, TN6-2 and TN6-3, were re-evaluated and compared to the parent strain TN5 in YP medium containing glucose and ethanol. The superior performance of TN6-1 was confirmed, producing $1130 \mathrm{mg} / \mathrm{L}$ muconic acid (31.42 mg/g carbon), in contrast to $555 \mathrm{mg} / \mathrm{L}$ muconic acid (16.68 mg/g carbon) for TN5 (Fig. 3B). The strains TN6-2 and TN6-3 also produced higher muconic acid concentrations than TN5, but not as pronounced as TN6-1. The residual PCA level was only $130 \mathrm{mg} / \mathrm{L}$ for the TN6-1 strain as opposed to $290 \mathrm{mg} / \mathrm{L}$ for the TN5 strain (Fig. 3B). All three TN6 isolates showed much slower glucose consumption rates than TN5, reflecting their lower glucose tolerance and utilization despite the presence of the MTH1 partial deletion and ethanol as $\mathrm{C} 2$ source in the medium. However, they all produced muconic acid faster than TN5. On the other hand, TN5 started to produce PCA faster than the TN6 strains and maintained a higher level (Fig. 3B). These results show that some of the TN6 strains with large colony size displayed a much higher flux in the pathway from glucose to muconic acid than the parent strain TN5. The TN5 strain had a low ethanol production (two copies of PDC5 were still present) and consumed the ethanol after the diauxic shift. The glucose and ethanol consumption rate of the three TN6 isolates was similar, indicating that it cannot be responsible for the superior production of muconic acid by TN6-1.

The five best performing isolates (TN6-1, TN6-2, TN6-3, TN6-4, TN6-5) were whole-genome sequenced to search for possible causative SNPs or other genomic modifications, different from the replacement of $P D C 5$ by MApw, responsible for their varying performance and in particular for the superior performance of TN6-1. The whole genome sequence of the TN5 strain served as reference. In the different TN6 transformants, we found changes in ploidy for several (parts of) chromosomes (Additional file 6: Table S1) and common (Additional file 7: Table S2) and unique (Additional file 8: Table S3) non-synonymous SNPs in many unrelated genes. Moreover, we observed that several TN6 transformants acquired SNPs in some MApw genes (Additional file 9: Table S4). The I218V SNP in the PCAD enzyme of TN6-1 stood out due to the increased conversion efficiency of PCA to muconic acid in this strain. Hence, the aro $Y$ - $C^{i s o}$, ${ }^{I 218 V}$ allele was reverted to its wild type form aroY-C $C^{\text {iso }}$ in the TN6-1 strain but this did not result in a different production profile, titer or yield for both PCA and muconic acid (Additional file 10). The sequencing data showed that the TN6 transformants displayed a considerable number of other SNPs and (partial) chromosomal ploidy changes that were difficult to disentangle in a straightforward way to investigate their possible involvement in the differences in performance and in particular the superior performance of strain TN6-1. 

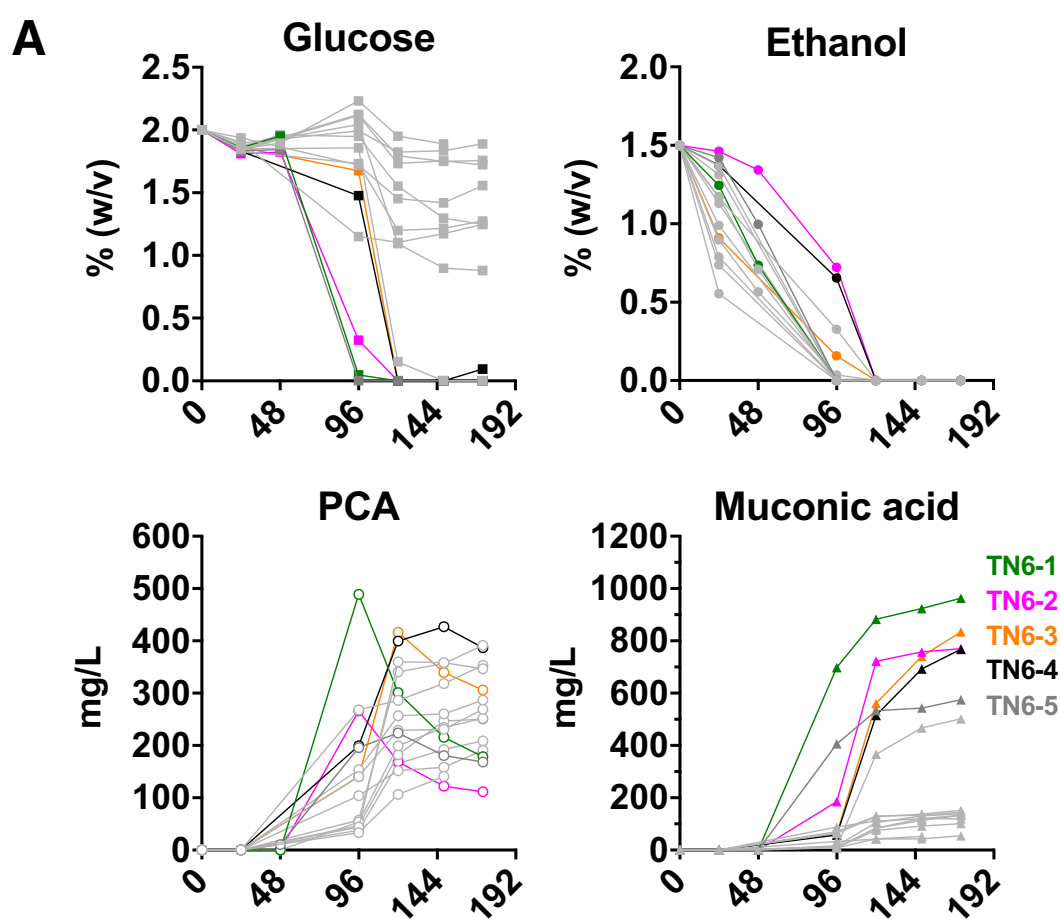

Time (h)

Time (h)

B
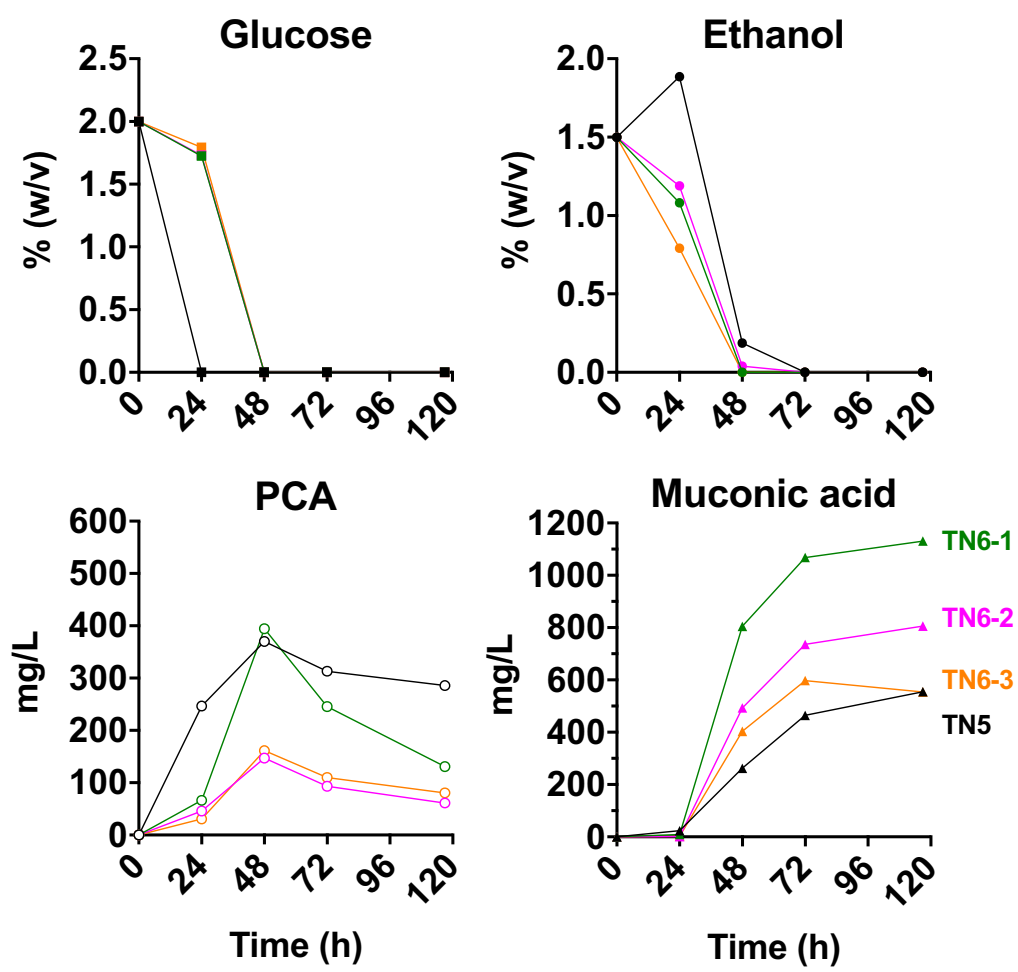

Fig. 3 PCA and muconic acid production by the TN5 strain and the TN6 isolates. YP2\%D1.6\%E medium was used. A Performance of all TN6 isolates tested. B Comparison of the three best isolates, TN6-1, TN6-2 and TN6-3, with parent strain TN5. Strains were inoculated at OD 600 0.1. TN5 (black), TN6-1 (green), TN6-2 (magenta), TN6-3 (orange) and other TN6-X isolates (grey) 
The use of a low pitching rate for TN6-1 under the obligatory aerobic conditions for muconic acid production led to very active propagation of the yeast resulting in a high loss of carbon into biomass formation instead of muconic acid production. For the next experiments, the pitching rate was therefore increased to $\mathrm{OD}_{600} 4( \pm 1.4 \mathrm{~g}$ $\mathrm{DW} / \mathrm{L}$ ). It resulted in higher muconic acid production and also in more complete xylose utilization (Additional file 11). A strong and sudden decrease in extracellular $\mathrm{pH}$ was also observed (Additional file 12), which we could partially counteract by the addition of a $50 \mathrm{mM}$ citrate buffer with an initial pH of 5.5 (Additional file 13). This also improved sugar consumption and muconic acid production. Although increasing the citrate buffer concentration to $100 \mathrm{mM}$ helped to increase the buffering capacity even further, it had an inhibitory effect on the fermentation (Additional file 14). We also evaluated higher inocula up to $\mathrm{OD}_{600} 32$ (Additional file 15) with $\mathrm{OD}_{600} 16( \pm 5.4 \mathrm{~g} \mathrm{DW} / \mathrm{L})$ resulting in the highest production of muconic acid.

Finally, the sequentially developed strains, T18HAA1 + pMApw, TN9, TN10, TN5 and TN6-1, were compared under the same conditions: YP medium buffered with $50 \mathrm{mM}$ citrate buffer at an initial $\mathrm{pH}$ of 5.5 , containing $2 \%$ glucose or $2 \%$ xylose supplemented with $0.5 \%$ ethanol and an initial pitching of $\mathrm{OD}_{600} 4$ (Fig. 4). There was a clear gradual increase in muconic acid production during strain development with the TN6-1 strain showing clearly superior performance. It reached a titer of $2350 \mathrm{mg} / \mathrm{L}$ with a yield of $53 \mathrm{mg} / \mathrm{g}$ carbon, in spite of its slower glucose and xylose consumption rate. It was the only strain without additional ethanol production. All strains still produced high levels of PCA, with TN6-1 the only strain showing a transient increase. The increase in the combined amount of PCA and muconic acid produced correlated with the increase in copy number of the genomically integrated MApw.

\section{Stimulation of PCA conversion to muconic acid in the TN6-1 strain}

Successful approaches to stimulate PCA conversion to muconic acid reported in literature include increasing the copy number of PCAD and related co-factor producing enzyme [25] or expressing co-factor
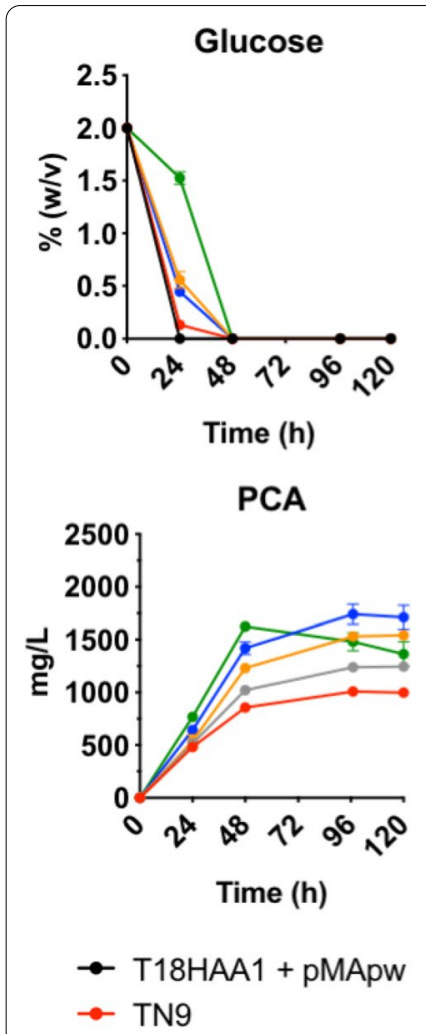
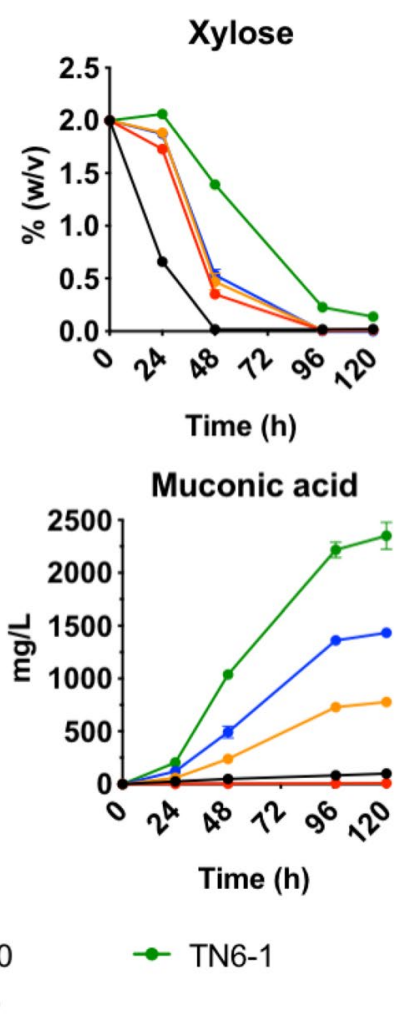

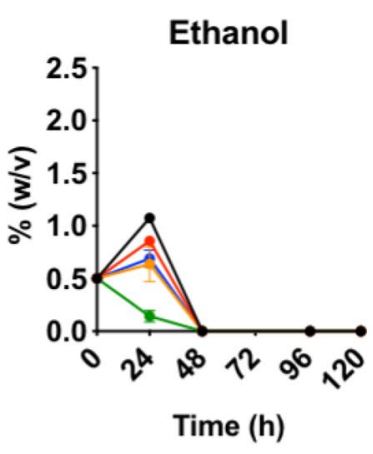

Yield

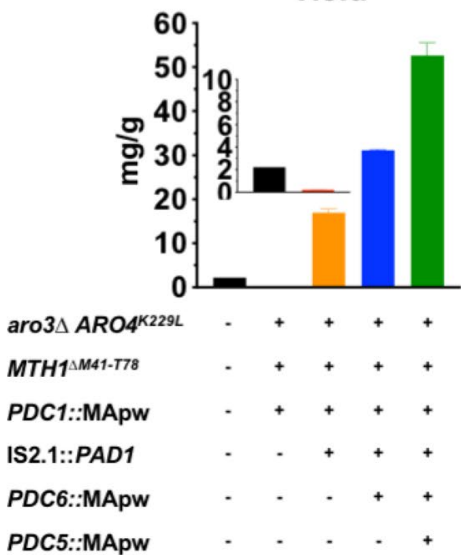

Fig. 4 Comparison of muconic acid fermentation performance in the sequentially constructed strains from T18HAA1 up to TN6-1. YP2\%D2\%X0.5\%E medium was used, buffered with $50 \mathrm{mM}$ citrate buffer at an initial pH of 5.5. Strains were inoculated at OD 600 of 4. Yield represents milligram of muconic acid produced per gram of initial carbon (glucose, xylose and ethanol), by the respective strains with corresponding genomic alterations indicated. Results are means of two biological replicates. Error bars show standard deviation at each time point 
independent PCAD enzymes [20]. We therefore integrated two additional copies of different heterologous genes, encoding PCAD, in the genome of TN6-1: the co-factor independent $A G D C 1$ from $A$. adeninivorans and TaGDC1 from T. atroroseus, as well as the co-factor dependent aroY- $C^{i s o}$ from K. pneumoniae. The constructs were inserted at the integration site IS16.2 of Chr. XVI (NC_001148.4:g.621736) yielding strains TN14, TN15 and TN22, respectively. To avoid possible feedback inhibition by muconic acid on the production rate, we used a low initial glucose concentration of $2.5 \%$ and addition of $0.5 \%$ ethanol as a C2 source. The TN14 and TN15 strains showed none or a slight improvement in the conversion of PCA, respectively, compared to the TN6-1 strain (Fig. 5A). However, in the TN22 strain, a strong reduction in PCA accumulation with a concomitant increase in muconic acid production was observed. This resulted in an enhanced yield of $62.3 \mathrm{mg} / \mathrm{g}$ carbon, compared to $44.6 \mathrm{mg} / \mathrm{g}$ carbon for TN6-1 (Fig. 5B).

\section{Maximal muconic acid production and toxicity of muconic acid on the TN22 strain}

Next, we tested the maximum titer of muconic acid that could be reached by the TN22 strain with glucose and xylose separately, and with a mixture of the two. To reduce the biomass formation we increased the pitching rate to an $\mathrm{OD}_{600}$ of $16( \pm 5.4 \mathrm{~g} \mathrm{DW} / \mathrm{L})$, since this was previously found to lead to the highest glucose consumption and muconic acid production (Additional file 15). The TN22 strain was inoculated in shake flasks with YP medium containing 10\% glucose (YPD), 10\% xylose (YPX) or $6 \%$ glucose and $5 \%$ xylose (YPDX) with the addition of $2 \%$ ethanol for all conditions. The medium was buffered with $50 \mathrm{mM}$ citrate buffer at a starting $\mathrm{pH}$ of 5.5. Muconic acid and PCA production were measured over time up to $144 \mathrm{~h}$ (Fig. 6). When glucose or xylose were used separately as carbon sources, both were converted into muconic acid, but glucose more efficiently, reaching titers of about $3700 \mathrm{mg} / \mathrm{L}$ and $2500 \mathrm{mg} / \mathrm{L}$, respectively. For xylose, the variation between replicates was larger, reaching $3400 \mathrm{mg} / \mathrm{L}$ in the best replicate (Fig. 6A). The production rate of muconic acid was higher with glucose compared to xylose, which resulted in a higher muconic acid yield with glucose than with xylose (Fig. 6B). When the medium contained a mixture of glucose and xylose, glucose was consumed preferentially over xylose, with poor consumption of the latter. In all cases, the extracellular $\mathrm{pH}$ dropped to 3.3 in $24 \mathrm{~h}$ and was maintained at around 3.5. Volumetric productivity was the highest at $48 \mathrm{~h}$ with glucose as the substrate $(37 \mathrm{mg} / \mathrm{L} \mathrm{h})$, followed by the mixture of glucose and xylose $(34.7 \mathrm{mg} / \mathrm{L} \mathrm{h})$ and then xylose $(32.3 \mathrm{mg} / \mathrm{L} \mathrm{h})$. Muconic acid titers were higher when glucose was present in the medium. The highest mean muconic acid titer was $3700 \mathrm{mg} / \mathrm{L}$ with glucose, then $3300 \mathrm{mg} / \mathrm{L}$ with a mixture of glucose and xylose, and $2600 \mathrm{mg} / \mathrm{L}$ with xylose.

In all cases, sugar consumption slowed down when muconic acid titers reached around $2000 \mathrm{mg} / \mathrm{L}$, and this was more pronounced for xylose. This suggested feedback inhibition by the increasing muconic acid level in the medium. Hence, we tested muconic acid toxicity using growth assays in media supplemented with various levels of muconic acid. It should be mentioned that the cis,cis muconic acid, the isomer that is produced during
A

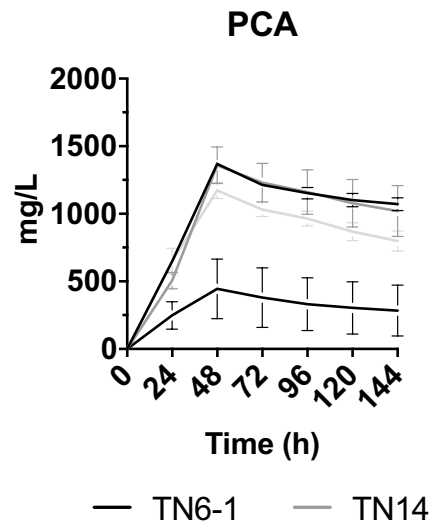

B

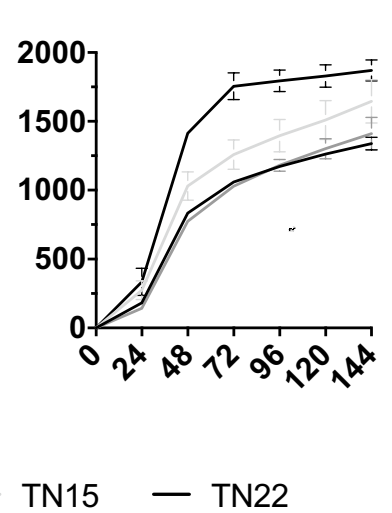

Yield

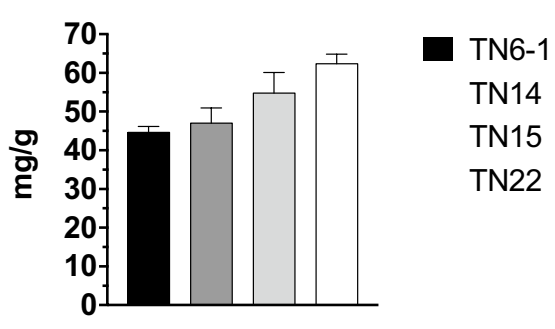

Fig. 5 PCA and muconic acid production by the TN6-1, TN14, TN15 and TN22 strains. A YP2.5\%D0.5\%E medium was used buffered with 50 mM citrate buffer at an initial pH of 5.5. Strains were inoculated at $\mathrm{OD}_{600}$ of 4 . Results are means of two biological replicates for TN6-1 and three independent isolates for the other strains. Error bars show standard deviation at each time point. B Yield of muconic acid (mg/g) from the fermentation shown in (A) at time point $144 \mathrm{~h}$ 


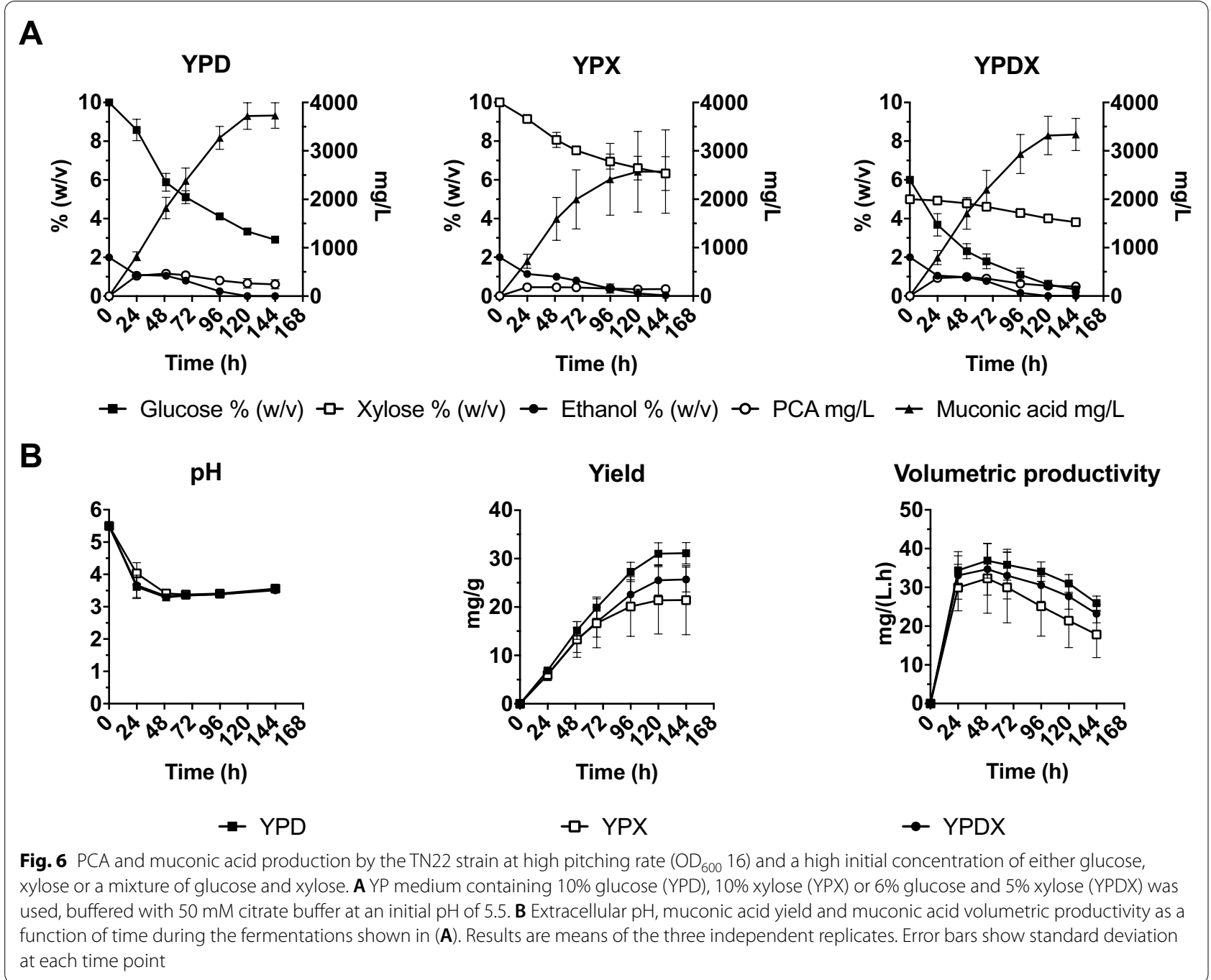

the fermentation, spontaneously isomerizes to cis,trans at $\mathrm{pH}$ values lower than 4 [4]. Moreover, when fully protonated, cis,cis muconic acid has a low solubility of $1 \mathrm{~g} / \mathrm{L}$ in water, while cis,trans muconic acid has a solubility of up to $5.2 \mathrm{~g} / \mathrm{L}$ [53]. The low final $\mathrm{pH}$ level, the temperature of fermentation $\left(30^{\circ} \mathrm{C}\right)$ and the high level of muconic acid obtained in our experiments suggested that under our conditions the produced cis,cis isomer spontaneously isomerized to the cis,trans isomer.

To test the toxicity, Dr. Ibrahim Khalil (CSCE, KU Leuven) provided a small amount of cis,cis muconic acid that was isomerized to cis,trans muconic acid. Different muconic acid concentrations of $0 \mathrm{mg} / \mathrm{L}, 2000 \mathrm{mg} / \mathrm{L}$ and $5000 \mathrm{mg} / \mathrm{L}$ were added to YP medium containing $10 \%$ glucose, $1 \%$ ethanol and $50 \mathrm{mM}$ citrate buffer. The initial $\mathrm{pH}$ was set to 4.4 instead of 5.5 , to mimic the lower $\mathrm{pH}$ level at the end of the fermentation where muconic acid seemed to be inhibitory, while allowing higher solubility of the cis,trans isomer. The TN22 strain was inoculated at an initial $\mathrm{OD}_{600} 0.1$ and $\mathrm{OD}_{600} 16$. In both cases, the presence of $2000 \mathrm{mg} / \mathrm{L}$ and $5000 \mathrm{mg} / \mathrm{L}$ lowered or severely compromised glucose consumption, muconic acid production and growth of the TN22 strain (Table 3).

Table 3 Determination of muconic acid toxicity on strain TN22

\begin{tabular}{ccclc}
\hline Initial $\mathbf{O D}_{600}$ & $\begin{array}{l}\text { MA } \\
\text { added } \\
\text { (mg/L) }\end{array}$ & $\begin{array}{l}\text { Glucose } \\
\text { consumed, \% } \\
(\mathbf{w} / \mathbf{v})\end{array}$ & $\begin{array}{l}\text { MA } \\
\text { produced }\end{array}$ & Final OD $_{600}$ \\
\hline 0.1 & 0 & $3.8( \pm 0.13)$ & $1660( \pm 46)$ & $24.3( \pm 1.2)$ \\
& 2000 & $3.5( \pm 0.16)$ & $870( \pm 33)$ & $24.0( \pm 1.0)$ \\
16 & 5000 & $0.09( \pm 0.004)$ & $29.7( \pm 29.7)$ & $7.9( \pm 0.1 .9)$ \\
& 0 & $6.9( \pm 0.08)$ & $3610( \pm 16.6)$ & $44.15( \pm 0.75)$ \\
& 2000 & $4.8( \pm 0.08)$ & $1100( \pm 47.1)$ & $35.1( \pm 0.4)$ \\
& 5000 & $2.4( \pm 0.1)$ & 0 & $30.4( \pm 0.3)$ \\
\hline
\end{tabular}


The TN22 strain was inoculated in YP10\%D2\%E medium with initial $\mathrm{pH} 4.4$ containing different levels of cis,trans muconic acid. Two different pitching rates of $\mathrm{OD}_{600} 0.1$ and $\mathrm{OD}_{600} 16$ were used and the fermentations were stopped after $137 \mathrm{~h}$ and $168 \mathrm{~h}$, respectively. Glucose consumption, muconic acid production and final $\mathrm{OD}_{600}$ were measured. Results show means and standard deviation of two biological replicates.

From the toxicity tests we can conclude that muconic acid is already exhibiting an inhibitory effect on the TN22 strain at titers of approximately $2900 \mathrm{mg} / \mathrm{L}(2000 \mathrm{mg} / \mathrm{L}$ added $+870 \mathrm{mg} / \mathrm{L}$ produced). Moreover, the muconic acid production was more sensitive to the presence of elevated muconic acid concentrations in the medium than the growth or glucose consumption of the yeast.

\section{In-situ extraction of muconic acid during batch fermentations}

To address the presumed feedback inhibition of muconic acid on its production, we explored in-situ product removal (ISPR) of the muconic acid during the fermentation. The Bio Base Europe Pilot Plant (Ghent, Belgium) performed a preliminary screening of various solvents and their biocompatibility for extraction of the muconic acid (see "Materials and methods" section). The screening identified polypropylene glycol 4000 (PPG) as an effective and biocompatible solvent for extraction of muconic acid, with a calculated distribution coefficient (Kd) of 5.78 .

We then tested the effect of adding PPG to the fermentation with the TN22 strain. The TN22 strain was inoculated at $\mathrm{OD}_{600} 16$ in shake flasks with YP medium containing $10 \%$ glucose, $10 \%$ xylose or a mixture of the two ( $6 \%$ glucose, $4.5 \%$ xylose), and $2 \%$ ethanol as a $\mathrm{C} 2$ source, buffered with $50 \mathrm{mM}$ citrate buffer at an initial $\mathrm{pH}$ of 5.5. The PPG was added at the beginning of the fermentation in a $1: 5$ ratio to the medium volume (i.e. $40 \mathrm{~mL}$ YP medium and $8 \mathrm{~mL}$ PPG). Under these conditions, the amount of muconic acid that accumulated in the fermentation medium was reduced by about $50 \%$ (Fig. 7B) compared to the control (Fig. 7A). To determine the amount of muconic acid in the PPG layer, we separated the layer from the aqueous phase and washed it with $4 \mathrm{~mL} 50 \%$ ethanol [54], and measured the muconic acid concentration in the ethanol using HPLC analysis. This showed that almost equal amounts of muconic acid could be recovered from the PPG phase compared to the amount present in the fermentation medium (Fig. 7C). In all cases, the titer and yield of the total amount of muconic acid produced were similar to those in the fermentation without PPG (Fig. 7C). In spite of the strong reduction by about $50 \%$ of the muconic acid titer in the fermentation medium in the presence of PPG, the sugar consumption still arrested after approximately half of the initial sugar concentration was utilized (Fig. 7B), similar to the fermentation in the absence of PPG (Fig. 7A). These results contradict that product inhibition by muconic acid is responsible or at least solely responsible for the arrest of sugar utilization. They suggest that another (possibly metabolic) bottleneck is building up during the production of muconic acid, which inhibits and finally stops the sugar consumption and muconic acid production.

The slow-down and subsequent arrest of sugar consumption cannot be due to a loss of the yeast's viability since we did not observe a pronounced drop in the number of colony forming units (CFU) up to $144 \mathrm{~h}$ in the fermentation with any of the sugar substrates. Only after $192 \mathrm{~h}$, and only in the fermentation with glucose, a strong drop in CFU was noticed, which was not affected by the presence of PPG (Additional file 16).

To confirm these conclusions, we repeated the muconic acid fermentations under the same conditions but with addition of PPG at the beginning of the fermentation in a 1:2 ratio to the medium volume (i.e. $40 \mathrm{~mL}$ YP medium and $20 \mathrm{~mL}$ PPG). This resulted in a further reduction of the muconic acid concentration in the medium and a concomitant increase in the PPG phase, but without any significant change in the sugar utilization pattern and total muconic acid production compared to the fermentations with a PPG to medium ratio of 1:5 (Additional file 17). The same observations were also made for the viability measurements. Only in the muconic acid production with glucose, there was a strong drop in CFUs late in the fermentation, which was not overcome by the presence of PPG. On the other hand, in the fermentations with xylose and mixed glucose and xylose, there was only a moderate drop in CFUs, which was also not affected by the presence of PPG (Additional file 18).

\section{Discussion}

The goal of the present work was to construct a secondgeneration yeast cell factory with efficient production of muconic acid from both glucose and xylose. We utilized the xylose-utilizing and inhibitor tolerant GSE16T18HAA1 as a starting strain [46]. Several known strategies are crucial in tailoring the central metabolism of $S$. cerevisiae to achieve high titers of muconic acid $[19-21,55]$. These all focus on increasing the DHS substrate availability by improving flux towards (TKL1 overexpression) and in the shikimate pathway (expression of $A R O 4^{K 229 L}$ and $A R O 3^{K 222 L}$ ) or lowering DHS breakdown towards aromatic amino acids $\left(A R O 1^{\text {aroEL }}\right)$. The starting strain GSE16-T18HAA1 used in this work overexpressed all the non-oxidative pentose phosphate pathway genes, including TKL1 [12], which was reported to boost E4P availability. The flux towards the shikimate pathway in 


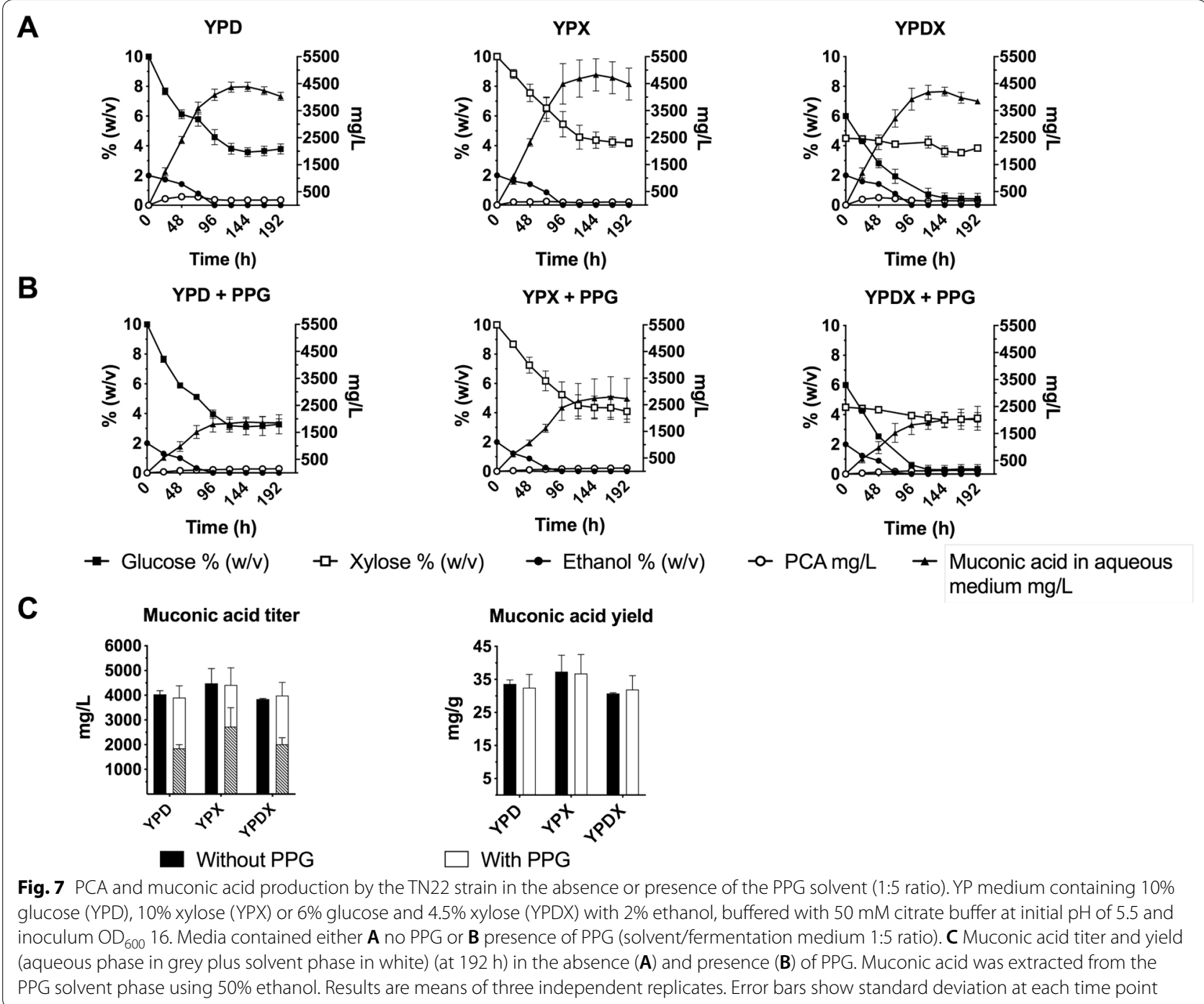

this strain was further increased by deleting $A R O 3$ and expressing the feedback resistant $A R O 4^{K 229 L}$. Overexpression of $A R O 4^{K 229 L}$ and $A R O 3^{K 222 L}$ has also been reported in the literature [20], but we were not able to obtain stable strains with these modifications. The choice for the heterologous genes used in this work is largely based on previous studies (Table 4), with exception of PCAD. We opted for the aroY-C $C^{i s o}$ isoform due to its reduced inhibition by oxygen [17]. Improvement of PCA conversion has been achieved by increasing the copy number of the gene encoding PCAD and its co-factor availability $[29,39,56]$. Despite the fact that the TN6 strain already expressed 6 copies of the gene encoding PCAD (2 copies in each of the $P D C$ loci) and had enhanced prFMN production due to insertion of two PAD1 overexpression cassettes, PCA still accumulated to high levels (Fig. 4). To address this problem we explored the additional expression of the prFMN-dependent enzymes aroY-C $C^{i s o}$ and the prFMN-independent PCAD enzymes ( $a G D C 1$ and TaGDC1) [20]. As opposed to the results in this previous work, we only observed significant improvement of PCA conversion resulting in higher muconic acid levels when we expressed two additional copies of the aroY-C $C^{\text {iso }}$ (Fig. 5). Thus, the level of prFMN does not seem to be a limiting factor for efficient muconic acid production in our strain background.

By applying these approaches and also eliminating ethanol production while increasing the copy number of the MApw, we were able to obtain a maximum titer of $4.5 \mathrm{~g} / \mathrm{L}$ muconic acid in batch fermentations with glucose and xylose. This is the highest titer reported in batch fermentations up to now (Table 4) and also the first report of muconic acid production without concomitant ethanol production.

The yeast $S$. cerevisiae has a very high capacity and preference for ethanol production [32]. No previous 
Table 4 Comparison of titer and yield for reported S. cerevisiae muconic acid cell factories

\begin{tabular}{|c|c|c|c|c|c|c|}
\hline Strain name & Genetic characteristics & Fermentation type (total h) & Carbon source & Titer (mg/L) & Yield (mg/g) & Refs \\
\hline CEN.PK2-1 CaroED & $A R O 1^{\text {aroEL }}$, aro $Z_{B t h}, a s b F_{B t h}$, aroY-Ciso & Batch (170 h) & $\mathrm{D}$ & 1.56 & 0.78 & [17] \\
\hline MuA12 & $\begin{array}{l}\operatorname{aro3} 3, z W f 1 \triangle, A R O 4^{K 229 L}, T K L 1 \\
\operatorname{aro}_{P a^{\prime}} \operatorname{aroY}_{E C^{\prime}} \mathrm{HQD}_{\mathrm{Ca}}\end{array}$ & Batch (108 h) & $\mathrm{D}$ & 141 & 3.53 & [21] \\
\hline MA4 & $\begin{array}{l}\operatorname{ric} 1 \triangle, \operatorname{aro1} 1 \triangle, A R O 4^{K 229 L}, \\
\quad A R O 1^{D 14091, D 920 A}, T K L 1, \text { aro } Z_{K p^{\prime}} \\
\operatorname{aroY}_{K p} H_{Q D 2} 2_{C a}\end{array}$ & Batch (72 h) & D & 2.7 & 33.57 & [23] \\
\hline MuA-5.01.1.02 & $\begin{array}{l}\text { TKL1, ARO }{ }^{\text {aroE }}, \text { aro }_{P a^{\prime}} \operatorname{aroY}_{E C^{\prime \prime}} \\
\quad H Q D 2_{C a}\end{array}$ & Fed-batch (240 h) & $\mathrm{D}$ & 2.1 & 12.9 & [24] \\
\hline JTY4 & 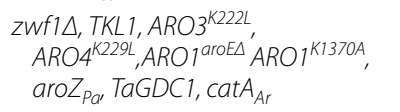 & Batch (120 h) & $\mathrm{D}$ & 1244 & 31 & [20] \\
\hline Isolate 7 & $\begin{array}{l}\text { TKL 1, aro } Z_{P a} \text { aro } Y-B, \text { aro } Y-C^{\text {iso }} \text {, aroY- } \\
D, \text { catA }_{C a}\end{array}$ & Fed-batch (120 h) & D & 2008 & 13.4 & [25] \\
\hline Aro $_{\text {PEST }}-\mathrm{Hqd} 2$ (p6G) & 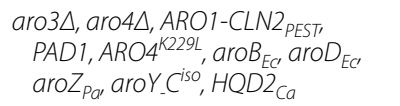 & Fed-batch (168 h) & D & 5.1 & 58 & {$[22]$} \\
\hline ST8943 & 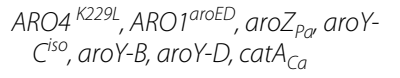 & Fed-batch (150 h) & D & 20,800 & 66.2 & [39] \\
\hline |c0.09-31 & $\begin{array}{l}\text { XK, PPP, ARO1 }{ }^{\text {aroed }}, X_{Y} \mid A_{B v} \text { aro } Z_{N C} \\
\operatorname{aroY}_{C i \nu} C a t A_{C n}\end{array}$ & Batch (72 h) & DX & 424 & 3.26 & [14] \\
\hline TN22 & $\begin{array}{l}\operatorname{aro} 3 \triangle, A R O 4^{K 229 L}, P A D 1, T K L 1 \\
\text { aro } Z_{P a \prime} \text { aroY-C } C^{\prime i s o}, H Q D 2_{C a}\end{array}$ & Batch (192 h) & ${ }^{\mathrm{a} D} / \mathrm{X} / \mathrm{DX}$ & $4030 / 4500 / 3840$ & 33.6/37.3/30.7 & This study \\
\hline
\end{tabular}

$D$ glucose, $X$ xylose

${ }^{\text {a }}$ Ethanol was added to the fermentation medium to address $C 2$ auxotrophy of the $p d c$-strain. Fermentations were performed in rich YP medium, buffered with citrate buffer at $\mathrm{pH} 5.5$ with an initial $\mathrm{OD}_{600} 16$

studies have focussed on completely eliminating ethanol production by deleting the $P D C$ genes for improving muconic acid production. Instead, they focussed on other approaches, such as lowering ethanol production by optimizing the feed composition and rate in fed-batch fermentations [39]. To eliminate ethanol production, we deleted the PDC1, 5 and 6 genes [34, 37, 57] and simultaneously integrated the MApw genes in these loci. Because of this approach it is difficult to determine to what extent the absence of ethanol production and/or the increased copy number of the MApw was responsible for the observed increase in muconic acid production.

Upon deleting the final $P D C$ gene, $P D C 5$, we obtained multiple transformants with highly varying performance. Of these transformants, TN6-1 showed the highest muconic acid production and the best conversion of PCA to muconic acid (Fig. 3). The differences in performance were likely caused by background mutations occurring in the transformants. In addition, pyruvate is known to accumulate in pdc-strains causing cytosolic acidification and a metabolic imbalance [32, 36]. Hence, the unknown mutations present in the TN6-1, TN6-2 and TN6-3 strains may lower the pyruvate accumulation for instance by increasing the conversion of pyruvate via PEP and E4P into the shikimate pathway causing higher muconic acid production. Because of the multiple genetic modifications present, we were so far unable to pinpoint the causative mutation(s) in these transformants (Additional files 7, 8, 9 and 10). Further detailed investigation, however, may likely reveal novel promising approaches to improve muconic acid production.

When we used higher initial sugar concentrations in order to obtain higher muconic acid titers, we observed stuck fermentations both with glucose and xylose, and a mixture of the two. The accumulation of about 3-4 g/L muconic acid always seemed to limit further production, irrespective of the level of PCA (Figs. 6A and 7A). Since we also found muconic acid to be toxic and strongly inhibiting at low $\mathrm{pH}$ its own production already at levels around $2 \mathrm{~g} / \mathrm{L}$ (Table 3), we envisaged the stuck muconic acid fermentations to be due to muconic acid toxicity. Previous work also reported muconic acid toxicity to be a limiting factor, especially at lower extracellular $\mathrm{pH}$ levels near the $\mathrm{p} K a$ (3.64) of muconic acid [39]. In that report, toxicity of the increasing concentrations of muconic acid was prevented by maintaining the extracellular $\mathrm{pH}$ at 6 in controlled fed-batch fermentations. This resulted in the more tolerable deprotonated form of muconic acid being the dominant form and allowed to reach a maximum titer of $20.8 \mathrm{~g} / \mathrm{L}$. An alternative or additional explanation is that the fed-batch process kept the sugar level continuously low so that the unknown bottleneck for 
muconic production that apparently built up in our strain during batch fermentation with high initial sugar levels, was never generated. In another study, Suastegui et al. also used high initial glucose concentrations of $8 \%$, which resulted in a muconic acid titer of $2.7 \mathrm{~g} / \mathrm{L}$ and an incomplete consumption of glucose with $2.5 \%$ left after $72 \mathrm{~h}$ in batch fermentation [23]. This fits with our observations that high initial sugar levels result in incomplete muconic acid fermentations.

Hence, we sought to further improve the muconic acid production by applying an ISPR approach using the PPG solvent, which could be essential for establishing a promising process for industrial application with the appropriate specifications for titer, productivity and yield. The addition of the PPG solvent resulted indeed in the efficient removal of approximately half (Fig. 7) or more than half (Additional file 17), depending on the PPG/medium volume ratio, of the muconic acid from the fermentation medium. However, in spite of the strong reduction in the muconic acid concentration in the fermentation medium, there was no improvement in sugar utilization and the fermentations remained stuck as in the absence of PPG. CFU measurements showed that this was not due to a loss in viability (Additional files 16, 18). The addition of PPG in a 1:2 ratio reduced the muconic acid concentration to levels between 500 and $1500 \mathrm{mg} / \mathrm{L}$ (Additional file 17). Such levels did not cause any inhibition of sugar utilization when lower initial sugar levels were used (Fig. 4, Additional files 10, 11, 12, 13 and 14). Hence, we are bound to conclude that during the muconic acid fermentation with high initial sugar levels an unidentified problem is generated, which starts to inhibit and finally completely blocks sugar consumption and muconic acid production. This could be a bottleneck in metabolism, such as a build-up of a metabolic intermediate, that starts to become toxic at high concentrations. Further research is needed to identify this specific problem and also whether it occurs in all strains or is related to the genetic modification introduced or to a mutation spontaneously generated in the TN22 strain. On the other hand, the similar result of incomplete sugar utilization reported by Suastegui et al. when using a high initial sugar concentration, suggest that it is independent of the genetic background and also of the pdc-genotype [23].

Although our approaches were successful in achieving a high titer of muconic acid both with glucose and xylose, there is still need for further substantial improvement. The following points can be addressed in future research.

First, detailed metabolomic analysis is required to identify the cause of the stuck muconic acid fermentations when high initial sugar levels are used. The latter are required to obtain high muconic acid titers. When the cause can be identified appropriate genetic modifications have to be introduced to overcome the problem. Alternatively, or when the problem cannot be identified in a straightforward manner, evolutionary adaptation could be explored to generate strains able to continue growth and sugar utilization over a longer time and thus outcompete the current strains that arrest growth and sugar utilization rapidly.

Second, although we had engineered an internal deletion in $M T H 1$, it only partially overcame the $\mathrm{C} 2$ requirement and ethanol addition was still necessary for efficient sugar consumption. Other approaches to address the C2 requirement have been proposed, but none of these fully overcame the $\mathrm{C} 2$ problem $[33,58]$. Evolutionary adaptation of the TN22 strain to obtain C2 independency might be more effective. At the same time the evolutionary adaptation might also lead to a higher muconic acid productivity.

Third, it is clear that muconic acid is toxic to S. cerevisiae, even at relatively low concentrations of 4-5 g/L especially at low $\mathrm{pH}$. This is important for muconic acid production with glucose, but even more with xylose, since xylose utilization is more sensitive to stress factors, like the presence of organic acids especially at low $\mathrm{pH}$ [59]. The xylose-utilization capacity of the TN22 strain was also reduced in the presence of glucose. This is a general problem with second-generation yeast strains [60]. To reach the high muconic acid titer, yield and productivity required for industrial implementation, ISPR of the muconic acid will be required. Our work has shown that PPG is a very effective solvent to remove muconic acid from the fermentation medium. Once the cause of the stuck fermentations has been addressed, application of ISPR with PPG may be highly effective in further increasing the maximal muconic acid titer obtained.

Fourth, the obligatory aerobic conditions result in significant biomass formation and loss of yield. Different approaches can be explored to reduce the growth capacity of the yeast under muconic acid production conditions.

\section{Conclusion}

We successfully constructed a second-generation yeast cell factory for the production of muconic acid with glucose and xylose as substrates. Ethanol production was completely eliminated and also production of the PCA intermediate was minimized. A maximal muconic acid titer of $4.5 \mathrm{~g} / \mathrm{L}$ was achieved, the highest obtained in batch fermentations with yeast up to now. We demonstrated toxicity of muconic acid in the concentration accumulated, for growth, sugar utilization and especially muconic acid production itself. High initial sugar levels resulted in stuck fermentations. Although ISPR with PPG reduced the muconic acid concentration in the medium 
to less than half, the arrest of sugar utilization and final drop in viability were not affected. This appears to indicate that an unknown, possibly metabolic bottleneck builds up during muconic acid fermentation with high sugar levels, which inhibits and finally terminates all further sugar utilization and muconic acid production.

\begin{abstract}
Abbreviations
YPD: Yeast extract peptone dextrose; PCA: Protocatechuic acid; MApw: Muconic acid biosynthesis pathway; DHS: 3-Dehydroshikimate; DHSD: DHS dehydratase; PCA: Protocatechuic acid; PCAD: PCA decarboxylase; CDO: Catechol 1,2-dioxygenase; PDC: Pyruvate decarboxylase; PEP: Phosphoenolpyruvate; E4P: Erythrose-4-phosphate; prFMN: Prenylated flavine mononucleotide; MTY: Maximum theoretical yield; CFU: Colony forming units; PPG: Polypropylene glycol 4000.
\end{abstract}

\section{Supplementary Information}

The online version contains supplementary material available at https://doi. org/10.1186/s12934-021-01594-3.

Additional file 1. PCA and muconic acid production by the TN7 strain expressing the MApw plasmid. Media containing (A) glucose, (B) xylose or (C) a mixture of glucose and xylose as substrate. Strains were inoculated at $\mathrm{OD}_{600} 1$. Results are the means of three independent replicates for each time point. Error bars show standard deviation at each time point.

Additional file 2. PCA and muconic acid production by the TN9 strain expressing two copies of the MApw at the PDC1 locus. Media containing (A) glucose, (B) xylose or (C) a mixture of glucose and xylose as carbon source. Strains were inoculated at $\mathrm{OD}_{600} 1$. Results are the means of three independent replicates for each time point. Error bars show standard deviation at each time point.

Additional file 3. PCA, catechol and muconic acid production by the TN8 pdc $1 \triangle$ strain expressing pMApw and either PAD1 or aro Y-B on multi-copy plasmids. Strains were inoculated in $\mathrm{YP} 4 \% \mathrm{D}$ medium and at $\mathrm{OD}_{600} 1$. Samples were taken after $48 \mathrm{~h}$. Inset shows in more detail the results for muconic acid.

Additional file 4. Production of PCA and muconic acid by the TN10 strain, which contains 2 copies of the MApw integrated in the PDC 1 locus and 2 copies of the PAD1 overexpression cassette. Media containing (A) glucose, (B) xylose or (C) a mixture of glucose and xylose as carbon source. Strains were inoculated at $\mathrm{OD}_{600} 1$. Results are the means of three independent replicates for each time point. Error bars show standard deviation at each time point.

Additional file 5. Production of PCA and muconic acid by the TN10 and TN5 strain, which contains two or four copies of the MApw, respectively. Strains were inoculated at $\mathrm{OD}_{600} 1$ and sampled after $48 \mathrm{~h}$ (A) and for TN5 also periodically during three days (B). YP containing $2 \%$ glucose and $2 \%$ xylose was used. Results are the means of two biological replicates for TN10 or three independent replicates for TN5. Error bars show standard deviation at each time point.

Additional file 6: Table S1. Ploidy of all chromosomes in the TN5 strain and the different TN6 transformants.

Additional file 7: Table S2. Common SNPs in the TN6 transformants compared to TN5.

Additional file 8: Table S3. Unique SNPs in each TN6 transformant compared to TN5.

Additional file 9: Table S4. SNPs in MApw genes in the TN6 transformants compared to TN5.

Additional file 10. PCA and muconic acid production by TN6-1 and TN16. (A) YP2.5\%D0.5\%E medium buffered with $50 \mathrm{mM}$ citrate buffer and initial $\mathrm{pH}$ of 5.5. Strains were inoculated at $\mathrm{OD}_{600}$ 4. Results are the means of two biological replicates for TN6-1 and three independent replicates for TN16. Error bars show standard deviation at each time point. (B) Total molar yield of PCA and muconic acid from fermentation experiment shown in (A) at time point $144 \mathrm{~h}$.

Additional file 11. Comparison of different pitching rates with TN6-1. YP2\%D2.25\%X1.6\%E medium. Results are means of two biological replicates. Error bars show standard deviation at each time point.

Additional file 12. Comparison of different glucose concentrations for the production of PCA and muconic acid by TN6-1. YP medium with 2\%, $5 \%$ or $10 \%$ glucose and $1.6 \%$ ethanol. Strains were inoculated at $\mathrm{OD}_{600} 4$. Results are means of two biological replicates. Error bars show standard deviation at each time point.

Additional file 13. Comparison of different glucose concentrations for the production of PCA and muconic acid with the TN6-1 strain in YP medium buffered with $50 \mathrm{mM}$ citrate buffer at initial $\mathrm{pH}$ of 5.5. YP medium with $2 \%, 5 \%$ or $10 \%$ glucose and $1.6 \%$ ethanol. Strains were inoculated at $\mathrm{OD}_{600} 4$. Results are means of two biological replicates. Error bars show standard deviation at each time point.

Additional file 14. Comparison of different glucose concentrations for the production of PCA and muconic acid by TN6-1 in YP medium buffered with $100 \mathrm{mM}$ citrate buffer at initial pH of 5.5. YP medium with $2 \%, 5 \%$ or $10 \%$ glucose and $1.6 \%$ ethanol. Strains were inoculated at $\mathrm{OD}_{600} 4$. Results are means of two biological replicates. Error bars show standard deviation at each time point.

Additional file 15. Comparison of different pitching rates for the production of PCA and muconic acid with the TN6-1 strain in YP medium buffered with $50 \mathrm{mM}$ citrate buffer at initial pH of 5.5. YP medium with 12\% glucose and $1.6 \%$ ethanol. Results are means of two biological replicates. Error bars show standard deviation at each time point.

Additional file 16. Determination of viability during muconic acid fermentations in YPD, YPX and YPDX. CFUs were determined at different time points during the fermentations with the TN22 strain in the absence or presence of the PPG solvent (1:5 ratio) (Fig. 7) up to $144 \mathrm{~h}$. They are shown on a logarithmic scale. (At $192 \mathrm{~h}$ very low and highly variable CFUs were found indicating strong loss of viability after extended incubation at the end of the fermentation.)

Additional file 17. PCA and muconic acid production by the TN22 strain in the presence of the PPG solvent (1:2 ratio). YP medium containing 10\% glucose (YPD), 10\% xylose (YPX) or $5 \%$ glucose and $5 \%$ xylose (YPDX) with $2 \%$ ethanol, buffered with $50 \mathrm{mM}$ citrate buffer at initial $\mathrm{pH}$ of 5.5 and inoculum $\mathrm{OD}_{600}$ 16. Media contained either (A) no PPG or (B) presence of PPG (solvent/fermentation medium 1:5 ratio). (C) Muconic acid titer and yield (aqueous phase in grey plus solvent phase in white) (at $216 \mathrm{~h}$ ) in the absence (A) and presence (B) of PPG. Muconic acid was extracted from the PPG solvent phase using 50\% ethanol. Results are means of three independent replicates. Error bars show standard deviation at each time point.

Additional file 18. Determination of viability during muconic acid fermentations in YPD, YPX and YPDX. CFUs were determined at different time points during the fermentations with the TN22 strain in the absence or presence of the PPG solvent (1:2 ratio) (Additional file 17) up to $216 \mathrm{~h}$. They are shown on a logarithmic scale.

\section{Acknowledgements}

We are grateful to Renata Wicik and Martine De Jonge for technical support and to Arne Claes, Quinten Deparis and Nico Vangoethem for help with preparation of figures and graphical abstract. JMT gratefully acknowledges the support by the Open Bio-Incubator in the Erasmus High School in Brussels (Jette).

\section{Authors' contributions}

JMT and MRF conceived the project, TN performed the experiments, TN, MRF and JMT analyzed the data, QD performed the bioinformatic analysis, and TN, MRF and JMT wrote the manuscript. All authors read and approved the final manuscript. 


\section{Funding}

This work was supported by SBO Grants 'ARBOREF' (IWT 140894) and 'SPICY' (HBC.2017.0597 from VLAIO/Catalisti) to JMT.

\section{Availability of data and materials}

All data have been stored on dedicated computers at KU Leuven. All data and yeast strains are freely available upon request. The sequencing data have been deposited with links to the BioProject Accession number PRJNA699459 in the NCBI BioProject database (https://www.ncbi.nlm.nih.giv/bioproject/).

\section{Declarations}

\section{Ethics approval and consent to participate}

Not applicable.

\section{Consent for publication}

Not applicable.

\section{Competing interests}

The authors declare no competing interests.

\section{Author details}

${ }^{1}$ Laboratory of Molecular Cell Biology, Institute of Botany and Microbiology, KU Leuven, Leuven-Heverlee, Belgium. ${ }^{2}$ Center for Microbiology, VIB, Kasteelpark Arenberg 31, 3001 Leuven-Heverlee, Flanders, Belgium. ${ }^{3}$ NovelYeast Bv, Open Bio-Incubator, Erasmus High School, Laarbeeklaan 121, 1090 Brussels (Jette), Belgium.

Received: 11 February 2021 Accepted: 11 May 2021

Published online: 07 June 2021

\section{References}

1. Averesch NJH, Kromer JO. Metabolic engineering of the shikimate pathway for production of aromatics and derived compounds-present and future strain construction strategies. Front Bioeng Biotechnol. 2018;6:32.

2. Dai Z, Nielsen J. Advancing metabolic engineering through systems biology of industrial microorganisms. Curr Opin Biotechnol. 2015;36:8-15.

3. Skoog E, Shin JH, Saez-Jimenez V, Mapelli V, Olsson L. Biobased adipic acid - the challenge of developing the production host. Biotechnol Adv. 2018;36:2248-63.

4. Khalil I, Quintens G, Junkers T, Dusselier D. Muconic acid isomers as platform chemicals and monomers in the biobased economy. Green Chem. 2020;22:1517-41.

5. Rorrer NA, Dorgan JR, Vardon DR, Martinez CR, Yang Y, Beckham GT. Renewable unsaturated polyesters from muconic acid. ACS Sustain Chem Eng. 2016;4:6867-76.

6. Matsumoto A, Matsumara T, Aoki S. Stereospecific polymerization of dialkyl muconates through free radical polymerization: isotropic polymerization and topochemical polymerization. Macromolecules 2016;29:426-32

7. Quintens G, Vrijsen JH, Adriaensens P, Vanderzande D, Junkers T. Muconic acid esters as bio-based acrylate mimics. Polym Chem. 2019;10:5555-63.

8. Pleissner D, Dietz D, van Duuren JB, Wittmann C, Yang X, Lin CS, Venus J. Biotechnological production of organic acids from renewable resources. Adv Biochem Eng Biotechnol. 2017;166:373-410.

9. Deparis Q, Claes A, Foulquié-Moreno MR, Thevelein JM. Engineering tolerance to industrially relevant stress factors in yeast cell factories. FEMS Yeast Res. 2017; 17:fox036

10. Robak K, Balcerek M. Review of second generation bioethanol production from residual biomass. Food Technol Biotechnol. 2018;56:174-87.

11. Brat D, Boles E, Wiedemann B. Functional expression of a bacterial xylose isomerase in Saccharomyces cerevisiae. Appl Environ Microbiol. 2009;75:2304-11.

12. Demeke MM, Dietz H, Li Y, Foulquie-Moreno MR, Mutturi S, Deprez S, Abt T, Bonini BM, Liden G, Dumortier F. Development of a D-xylose fermenting and inhibitor tolerant industrial Saccharomyces cerevisiae strain with high performance in lignocellulose hydrolysates using metabolic and evolutionary engineering. Biotechnol Biofuels. 2013;6:1-24.
13. Zhang H, Pereira R, Li Z, Stephanopoulos G. Engineering Escherichia coli coculture systems for the production of biochemical products. Proc Natl Acad Sci USA. 2015;112:8266-71.

14. Liu T, Peng B, Huang S, Geng A. Recombinant xylose-fermenting yeast construction for the co-production of ethanol and cis, cismuconic acid from lignocellulosic biomass. Bioresour Technol Rep. 2020;9:100395.

15. Bui V, Lau KM, MacRea D, Schweitzer D. Methods for producing isomers of muconic acid and muconate salts. US patent 8,809,583 B2. 2014

16. Sauer M, Porro D, Mattanovich D, Branduardi P. Microbial production of organic acids: expanding the markets. Trends Biotechnol. 2008:26:100-8.

17. Weber C, Bruckner C, Weinreb S, Lehr C, Essl C, Boles E. Biosynthesis of cis, cis-muconic acid and its aromatic precursors, catechol and protocatechuic acid, from renewable feedstocks by Saccharomyces cerevisiae. Appl Environ Microbiol. 2012;78:8421-30.

18. Cao M, Gao M, Suastegui M, Mei Y, Shao Z. Building microbial factories for the production of aromatic amino acid pathway derivatives: from commodity chemicals to plant-sourced natural products. Metab Eng. 2020:58:94-132.

19. Hartmann M, Scheider TR, Pfeil A, Heinrich G, Lipscomb WN, Braus GH Evolution of feedback-inhibited beta/alfa barrel isoenzymes by gene duplication and a single mutation. Proc Natl Acad Sci. 2003;100:862-7.

20. Bruckner C, Oreb M, Kunze G, Boles E, Tripp J. An expanded enzyme toolbox for production of cis, cis-muconic acid and other shikimate pathway derivatives in Saccharomyces cerevisiae. FEMS Yeast Res. 2018;18:foy017.

21. Curran KA, Leavitt JM, Karim AS, Alper HS. Metabolic engineering of muconic acid production in Saccharomyces cerevisiae. Metab Eng. 2013;15:55-66.

22. Pyne ME, Narcross L, Melgar M, Kevvai K, Mookerjee S, Leite GB, Martin VJJ. An engineered Aro1 protein degradation approach for increased cis, cis-muconic acid biosynthesis in Saccharomyces cerevisiae. Appl Environ Microbiol. 2018. https://doi.org/10.1128/AEM.01095-18.

23. Suastegui M, Yu Ng C, Chowdhury A, Sun W, Cao M, House E, Maranas $C D$, Shao Z. Multilevel engineering of the upstream module of aromatic amino acid biosynthesis in Saccharomyces cerevisiae for high production of polymer and drug precursors. Metab Eng. 2017;42:134-44.

24. Leavitt JM, Wagner JM, Tu CC, Tong A, Liu Y, Alper HS. Biosensor-enabled directed evolution to improve muconic acid production in Saccharomyces cerevisiae. Biotechnol J. 2017;12:1600687.

25. Snoek T, Romero-Suarez D, Zhang J, Ambri F, Skjoedt ML, Sudarsan S, Jensen MK, Keasling JD. An orthogonal and pH-tunable sensor-selector for muconic acid biosynthesis in yeast. ACS Synth Biol. 2018;7:995-1003.

26. Payne KA, White MD, Fisher K, Khara B, Bailey SS, Parker D, Rattray NJ, Trivedi DK, Goodacre R, Beveridge R, et al. New cofactor supports alpha, beta-unsaturated acid decarboxylation via 1,3-dipolar cycloaddition. Nature. 2015;522:497-501.

27. Suastegui M, Matthiesen JE, Carraher JM, Hernandez N, Quiroz NR, Okerlund A, Cochran EW, Shao Z, Tessonnier JP. Combining metabolic engineering and electrocatalysis: application to the production of polyamides from sugar. Angew Chem. 2016;55:2368-73.

28. Weber HE, Gottardi M, Bruckner C, Oreb M, Boles E, Tripp J. Requirement of a functional flavin mononucleotide prenyltransferase for the activity of a bacterial decarboxylase in a heterologous muconic acid pathway in Saccharomyces cerevisiae. Appl Environ Microbiol. 2017. https://doi.org/ 10.1128/AEM.03472-16.

29. Skjoedt ML, Snoek T, Kildegaar KR, Arsovska D, Eichenberger M, Goedecke TJ, Rajkumar AS, Zhang J, Kristensen M, Lehka BJ, et al. Engineering prokaryotic transcriptional activators as metabolite biosensors in yeast. Nat Chem Biol. 2016:12:951-8.

30. Meier AK, Worch S, Boer E, Hartmann A, Mascher M, Marzec M, Scholz U, Riechen J, Baronian K, Schauer F, et al. Agdc1p—a gallic acid decarboxylase involved in the degradation of tannic acid in the yeast Blastobotrys (Arxula) adeninivorans. Front Microbiol. 2017;8:1777.

31. Flikweert MT, Van Der Zanden L, Janssen WM, Steensma HY, Van Dijken JP, Pronk JT. Pyruvate decarboxylase: an indispensable enzyme for growth of Saccharomyces cerevisiae on glucose. Yeast. 1996;12:247-57.

32. Pronk J, Steensma HY, van Dijken JP. Pyruvate metabolism in Saccharomyces cerevisiae. Yeast. 1996;12:1607-33. 
33. Dai Z, Huang M, Chen Y, Siewers V, Nielsen J. Global rewiring of cellular metabolism renders Saccharomyces cerevisiae Crabtree negative. Nat Commun. 2018;9:3059.

34. Kim SJ, Seo SO, Jin YS, Seo JH. Production of 2,3-butanediol by engineered Saccharomyces cerevisiae. Bioresour Technol. 2013;146:274-81.

35. Oud B, Flores CL, Gancedo C, Zhang X, Trueheart J, Daran JM, Pronk JT, van Maris AJ. An internal deletion in MTH1 enables growth on glucose of pyruvate-decarboxylase negative, non-fermentative Saccharomyces cerevisiae. Microb Cell Fact. 2012;11:131.

36. van Maris AJ, Geertman JM, Vermeulen A, Groothuizen MK, Winkler AA, Piper MD, van Dijken JP, Pronk JT. Directed evolution of pyruvate decarboxylase-negative Saccharomyces cerevisiae, yielding a C2-independent glucose-tolerant, and pyruvate-hyperproducing yeast. Appl Environ Microbiol. 2004;70:159-66.

37. Yu T, Zhou YJ, Huang M, Liu Q, Pereira R, David F, Nielsen J. Reprogramming yeast metabolism from alcoholic fermentation to lipogenesis. Cell. 2018;174:1549-1558.e1514.

38. Zhang Y, Liu G, Engqvist MK, Krivoruchko A, Hallstrom BM, Chen Y, Siewers $\checkmark$, Nielsen J. Adaptive mutations in sugar metabolism restore growth on glucose in a pyruvate decarboxylase negative yeast strain. Microb Cell Fact. 2015;14:116.

39. Wang G, Ozmerih S, Guerreiro R, Meireles AC, Carolas A, Milne N, Jensen MK, Ferreira BS, Borodina I. Improvement of cis, cis-muconic acid production in Saccharomyces cerevisiae through biosensor-aided genome engineering. ACS Synth Biol. 2020;9:634-46.

40. Lopez-Garzon CS, Straathof AJ. Recovery of carboxylic acids produced by fermentation. Biotechnol Adv. 2014;32:873-904.

41. Yoshikawa N, Mizuno S, Ohta K, Suzuki M. Microbial production of cis, cis-muconic acid. J Biotechnol. 1990;14:203-10.

42. Kertes AS, King CJ. Extraction chemistry of fermentation product carboxylic acids. Biotechnol Bioeng. 1986;28:269-82.

43. Canari R, Eyal AM. Effect of $\mathrm{pH}$ on dicarboxylic acids in extraction by amine-based extractants. Ind Eng Chem Res. 2003;42:1293-300.

44. Canari R, Eyal AM. Extraction of carboxylic acids by amine-based extractants: apparent extractant basicity according to the $\mathrm{pH}$ of half-neutralization. Ind Eng Chem Res. 2003;42:1285-92.

45. Gorden J, Zeiner T, Brandenbusch C. Reactive extraction of cis, cismuconic acid. Fluid Phase Equilib. 2015;393:78-84

46. Meijnen JP, Randazzo P, Foulquie-Moreno MR, van den Brink J, Vandecruys P, Stojiljkovic M, Dumortier F, Zalar P, Boekhout T, Gunde-Cimerman N, et al. Polygenic analysis and targeted improvement of the complex trait of high acetic acid tolerance in the yeast Saccharomyces cerevisiae. Biotechnol Biofuels. 2016;9:5.

47. Wallace-Salinas V, Signori L, Li Y-Y, Ask M, Bettiga M, Porro D, Thevelein JM, Branduardi P, Foulquie-Moreno MR, Gorwa-Grauslund MF. Reassesment of YAP1 and MCR1 contributions to inhibitor tolerance in robust engineered Saccharomyces cerevisiae fermenting undetoxified lignocellulosic hydrolysate. AMB Express. 2014. https://doi.org/10.1186/ s13568-014-0056-5.

48. Benatuil L, Perez JM, Belk J, Hsieh CM. An improved yeast transformation method for the generation of very large human antibody libraries. Protein Eng Des Sel. 2010;23:155-9.

49. Gietz RD, Schiestl RH. High-efficiency yeast transformation using the LiAc/SS carrier DNA/PEG method. Nat Protoc. 2007;2:31-4.

50. Langmead B, Salzberg SL. Fast gapped-read alignment with Bowtie 2. Nat Methods. 2012:9:357-9.

51. Li H, Handsaker B, Wysoker A, Fennell T, Ruan J, Homer N, Marth G, Abecasis G, Durbin R, Genome Project Data Processing S. The Sequence Alignment/Map format and SAMtools. Bioinformatics. 2009;25:2078-9.

52. Duitama J, Quintero JC, Cruz DF, Quintero C, Hubmann G, FoulquieMoreno MR, Verstrepen KJ, Thevelein JM, Tohme J. An integrated framework for discovery and genotyping of genomic variants from highthroughput sequencing experiments. Nucleic Acids Res. 2014;42:e44.

53. Carraher JM, Matthiesen JE, Tessonnier J-P. Comments on "Thermodynamics of cis, cis-muconic acid solubility in various polar solvents at low temperature range." J Mol Liq. 2016;224:420-2.

54. Scelfo S, Pirone R, Russo N. Thermodynamics of cis, cis-muconic acid solubility in various polar solvents at low temperature range. J Mol Liq. 2016;222:823-7.

55. Suastegui M, Guo W, Feng X, Shao Z. Investigating strain dependency in the production of aromatic compounds in Saccharomyces cerevisiae. Biotechnol Bioeng. 2016;113:2676-85.

56. Sonoki T, Morooka M, Sakamoto K, Otsuka Y, Nakamura M, Jellison J, Goodell B. Enhancement of protocatechuate decarboxylase activity for the effective production of muconate from lignin-related aromatic compounds. J Biotechnol. 2014:192 Pt A:71-7.

57. Zhou YJ, Buijs NA, Zhu Z, Qin J, Siewers V, Nielsen J. Production of fatty acid-derived oleochemicals and biofuels by synthetic yeast cell factories. Nat Commun. 2016;7:11709.

58. Kim JW, Kim J, Seo SO, Kim KH, Jin YS, Seo JH. Enhanced production of 2,3-butanediol by engineered Saccharomyces cerevisiae through fine-tuning of pyruvate decarboxylase and NADH oxidase activities. Biotechnol Biofuels. 2016;9:265.

59. Bellissimi E, van Dijken JP, Pronk JT, van Maris AJ. Effects of acetic acid on the kinetics of xylose fermentation by an engineered, xylose-isomerasebased Saccharomyces cerevisiae strain. FEMS Yeast Res. 2009;9:358-64.

60. Ko JK, Lee SM. Advances in cellulosic conversion to fuels: engineering yeasts for cellulosic bioethanol and biodiesel production. Curr Opin Biotechnol. 2018:50:72-80.

\section{Publisher's Note}

Springer Nature remains neutral with regard to jurisdictional claims in published maps and institutional affiliations.
Ready to submit your research? Choose BMC and benefit from:

- fast, convenient online submission

- thorough peer review by experienced researchers in your field

- rapid publication on acceptance

- support for research data, including large and complex data types

- gold Open Access which fosters wider collaboration and increased citations

- maximum visibility for your research: over 100M website views per year

At BMC, research is always in progress.

Learn more biomedcentral.com/submissions 